Draft version September 21, 2021

Preprint typeset using $\mathrm{L}_{\mathrm{A}} \mathrm{EX}$ style emulateapj v. 5/2/11

\title{
PROBING THE SOLAR WIND ACCELERATION REGION WITH THE SUN-GRAZING COMET C/2002 S2
}

\author{
S. Giordano ${ }^{1}$, J. C. Raymond ${ }^{2}$, P. LAmY ${ }^{3}$, M. Uzzo $^{4}$ And D. DobrzyckA ${ }^{5}$ \\ Draft version September 21, 2021
}

\begin{abstract}
Comet C/2002 S2, a member of the Kreutz family of Sungrazing comets, was discovered in white light images of the SOHO/LASCO coronagraph on 2002 September 18 and observed in H I Ly $\alpha$ emission by the SOHO/UVCS instrument at four different heights as it approached the Sun. The H I Ly $\alpha$ line profiles detected by UVCS are analyzed to determine the spectral parameters: line intensity, width and Doppler shift with respect to the coronal background. Two dimensional comet images of these parameters are reconstructed at the different heights. A novel aspect of the observations of this sungrazing comet data is that, whereas the emission from the most of the tail is blue-shifted, that along one edge of the tail is red-shifted. We attribute these shifts to a combination of solar wind speed and interaction with the magnetic field. In order to use the comet to probe the density, temperature and speed of the corona and solar wind through which it passes, as well as to determine the outgassing rate of the comet, we develop a Monte Carlo simulation of the H I Ly $\alpha$ emission of a comet moving through a coronal plasma. From the outgassing rate, we estimate a nucleus diameter of about 9 meters. This rate steadily increases as the comet approaches the Sun while the optical brightness decreases by more than a factor of ten and suddenly recovers. This indicates that the optical brightness is determined by the lifetimes of the grains, sodium atoms and molecules produced by the comet.

Subject headings: comets: general - comets: individual (C/2002S2) - Sun: corona - Sun: solar wind - ultraviolet: general
\end{abstract}

\section{INTRODUCTION}

Over 2000 comets have been discovered by the LASCO coronagraphs aboard the SOHO spacecraft, most of them members of the Kreutz family of sungrazing comets. These comets follow similar orbits, with perihelion close to the surface of the Sun, so few survive the encounter, a notable exception being Comet Lovejoy (C/2011 W3) (McCauley et al. 2013; Downs et al. 2013; Raymond et al. 2014). The Kreutz family is believed to come from the breakup of a single progenitor at least 1700 years in the past (Sekanina \& Chodas 2004; Marsden 2005), and close pairs of comets indicate that breakup occurs throughout the orbit (Sekanina \& Chodas 2007).

The LASCO observations show a consistent pattern of brightness as a function of distance from the Sun. The comets increase rapidly in brightness until they reach about $12 R_{\odot}$, at which point they begin to fade rapidly, presumably because the dust that scatters visible light begins to sublimate rapidly (Biesecker et al. 2002). The brightest ones are seen to level off at a lower brightness at smaller radii, though the brightness fluctuates. This may be a sign that the nucleus is breaking up.

The UVCS instrument aboard SOHO has obtained ultraviolet spectra of a number of Kreutz family comets.

\footnotetext{
${ }^{1}$ INAF-Osservatorio Astrofisico di Torino, via Osservatorio 20, 10025 Pino Torinese, Italy

${ }^{2}$ Harvard-Smithsonian Center for Astrophysics, 60 Garden St. Cambridge, MA 02138, USA

${ }^{3}$ Laboratoire d'Astrophysique de Marseille, 38 rue Frédéric Joliot-Curie, 13388 Marseille cedex 13, France.

${ }^{4}$ Computer Science Corporation, 3700 San Martin Drive, Baltimore, MD 21218 USA

${ }^{5}$ European Southern Observatory, Karl Schwarzschild Strasse 2, 85748 Garching (Germany)
}

Water outgassing from the comet is rapidly photodissociated. The resulting hydrogen atoms can scatter $\operatorname{Ly} \alpha$ photons from the solar disk, but their motion toward the Sun Doppler shifts the scattering cross section profile away from the solar emission line profile (Doppler dimming, also known as the Swings effect). Therefore, most of the observed Ly $\alpha$ comes from $\mathrm{H}$ atoms that have gone through charge transfer with the ambient coronal or solar wind protons, and they have a velocity distribution similar to that of the proton thermal distribution. We refer to the neutral populations before and after charge transfer as first and second generation hydrogen atoms, respectively.

The UVCS spectra have been used to determine the solar wind speed at $6.8 R_{\odot}$ in a coronal hole from the line width and the conditions for the comet bow shock (Ravmond et al. 1998). They imply nucleus sizes on the order of $10 \mathrm{~m}$ (Ravmond et al. 1998; Uzzo \& Marsden 2002; Bemporad et al. 2005) to hundreds of meters (McCauley et al. 2013). Increases in the apparent size of the nucleus imply breakup, providing an estimate of the tensile strength of the nucleus (Uzzo \& Marsden 2002). The time scale for fading of the Ly $\alpha$ brightness makes it possible to determine the coronal density without integration along a line-of-sight (Uzzo et al. 2001). A persistent Ly $\alpha$ signature can be interpreted in terms of a refractory population of grains (Kimura et al. 2002; Bemporad et al. 2005). UVCS observations of C III and $\mathrm{Si}$ III lines from a bright sungrazer indicate an overabundance of $\mathrm{Si}$ compared to $\mathrm{C}$ in the cometary dust (Ciaravella et al. 2010).

The comet $\mathrm{C} / 2002 \mathrm{~S} 2$ is a bright member of the Kreutz family discovered on 18 September, 2002. It reached an apparent $\mathrm{V}$ magnitude of 3.3 , then faded suddenly by 
over an order of magnitude in the optical when it reached a height of $5.7 R_{\odot}$, then recovered just as rapidly. Meanwhile, the Ly $\alpha$ brightness observed by UVCS increased steadily as the optical faded. In this comet we detect Ly $\alpha$ from both pre- and post-charge transfer hydrogen atoms, providing additional constraints on the outgassing rate and the coronal parameters.

The most remarkable feature of the observations is a substantial blue-shift of the northern part of the tail and red-shift of the southern part. This requires some mechanism to break the symmetry of the coronal velocity distribution or the charge transfer process. We suggest that the blue-shift is the line-of-sight component of the solar wind, while the red-shift results from pickup ion process similar to that studied in Comet Lovejoy by Raymond et al. (2014). When an atom is ionized it becomes a pickup ion with velocity components parallel and perpendicular to the magnetic field, and in this case the parallel component is away from the Earth. Subsequent charge transfer events with other neutrals produce a red-shifted population of hydrogen atoms moving in the direction of the magnetic field.

We construct a three-dimensional time-dependent Monte Carlo model of the kinematics (trajectory) of the outgassed neutral hydrogen, taking into account the ionization and charge transfer processes and use it to infer the outgassing rates and coronal parameters, such as outflow wind velocity, electron density and proton temperature. While most remote sensing observations provide only line-of-sight integrated quantities and averages, the comet allows us to probe individual points along the comet's path. Section 2 describes the observations and comet kinematics. In Section 3 we present an overview of the physical processes involved in the Ly $\alpha$ emission, Section 4 describes the Monte Carlo simulations and comparison with observation, and Section 5 discusses the derived comet and coronal parameters and the discrepancies between model and observation.

\section{OBSERVATIONS}

\subsection{LASCO Observations and Comet Kinematics}

The comet C/2002 S2 was discovered in LASCO data on 18 September, 2002 at a heliocentric distance larger that $16 R_{\odot}$ and it was followed down to about $3.0 R_{\odot}$, where it progressively disappeared and apparently sublimated before perihelion. The two coronagraphs involved in the observation are $\mathrm{C} 2$ and C3. A clear filter was used for C3 observation, with a nominal bandpass of 4000 to $8500 \AA$, while $\mathrm{C} 2$ observes with an orange filter, which selects the bandpass from 5400 to $6400 \AA$. For a detailed description of LASCO system, see Brueckner et al. (1995).

The comet approaches the Sun from the southwest at a position angle of about $236^{\circ}$ counterclockwise from north pole. In Figure 1 we show the LASCO C2 image taken on September 18, 2002 at 21:30 UT, when the comet was at the heliocentric distance of about $6.9 R_{\odot}$ projected into the plane of the sky; in the same figure we show a detail of the comet image where the comet tail is clearly visible. The visible length of the tail is real because in the 25 second exposure time, the comet, moving in the plane of the sky at a velocity of about $200 \mathrm{~km} \mathrm{~s}^{-1}$ covers a spatial region smaller than the $\mathrm{C} 2$ pixel size, which is 11.9 arcsec. The orbital parameters released in the Mi-

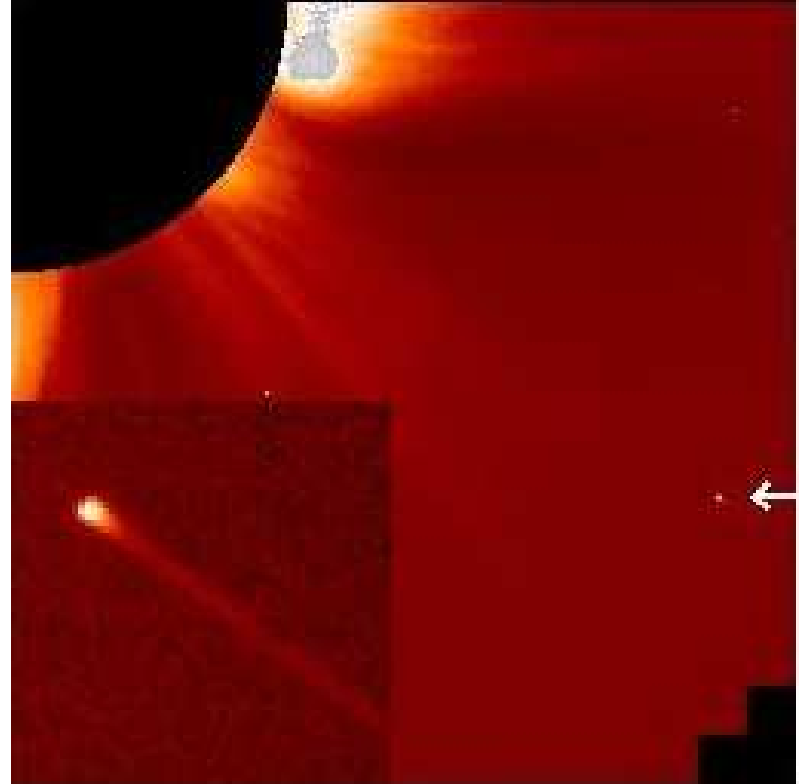

FIG. 1. - LASCO C2 comet C/2002 S2 observation on September 18,2002 at 21:30 UT. In the bottom left inset a zoomed image of the comet is shown.

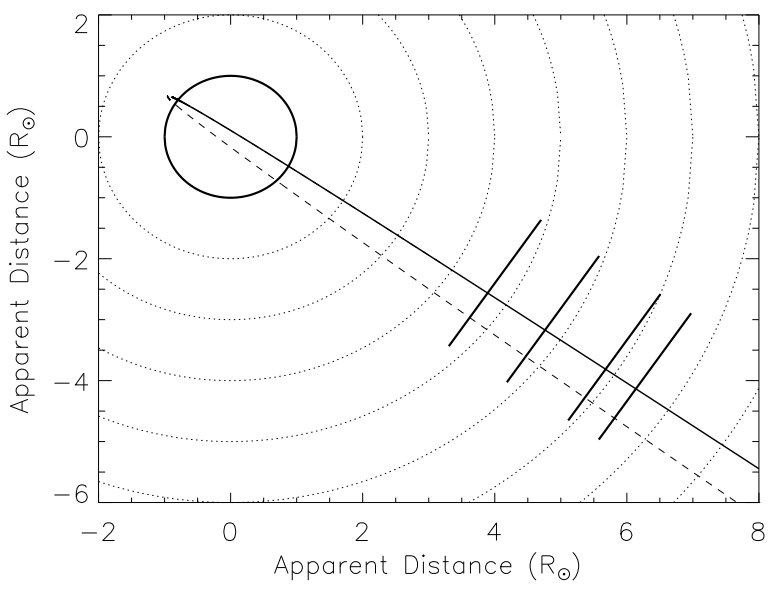

FIG. 2.- Comet C/2002 S2 trajectory in the plane of the sky as computed from the orbital parameters. We plot both the preperihelion (solid line) and the expected post-perihelion (dashed line). The UVCS slit positions at the observed heliocentric distances are also shown by the solid lines perpendicular to the trajectory.

nor Planet Electronic Circular issued on September 18, 2002 (Uzzo \& Marsden 2002) made it possible to adjust the UVCS pointing in order to detect the comet's ultraviolet emission and a subsequent more precise comet ephemeris computation MPEC 2002-S36 (see Table 1) permits a more accurate comet orbit computation and therefore the determination of the position and the kinematic parameters of the comet.

The comet orbit projected into the plane of the sky is shown in Figure 2 with the UVCS slit positions superimposed. Table 2 shows the comet position and kinematic parameters, computed from ephemeris, at the time of the first UVCS comet detection at each observed height, $t_{\text {enter }}$, given by the average time between the beginning 
TABLE 1

Comet C/2002 S2 orbital Elements

\begin{tabular}{|c|c|l|}
\hline Element & Value & Description \\
\hline$T_{\text {pass }}$ & 2002 Sept. 19.12 TT & Time of perihelion passage \\
$q$ & $0.0053 \mathrm{AU}$ & Perihelion distance \\
Per $i=\omega$ & $81.07^{\circ}$ & Argument of perihelion \\
Node $=\Omega$ & $3.76^{\circ}$ & Longitude of the ascending node \\
Incl $=i$ & $144.31^{\circ}$ & Inclination \\
$\epsilon$ & 1.0 & Eccentricity \\
\hline
\end{tabular}

and the end of the UVCS exposure which first detected the comet in $\mathrm{H}$ I Ly $\alpha$. The actual heliocentric distance of the comet from the Sun is given by $r$, and $\rho_{o b s}$ is the comet-Sun distance in the plane of the sky, that is the observed height. The comet is moving toward the Sun with a phase angle, $\alpha$, ranging from $\approx 30$ to $40^{\circ}$, which is the angular distance of the comet from the plane of the sky toward the Earth. We verify that the expected positions projected into the plane of the sky from computation agree well with the observed positions by LASCO and UVCS. In Table 2 we also show for each height the number of UVCS exposures detecting comet and tail in column $N_{\text {exp }}$ and the peak of the measured H i Ly $\alpha$ flux, $\mathrm{F}_{\alpha}$, in photons $\mathrm{cm}^{-2} \mathrm{~s}^{-1}$ (see Section 2.2).

TABLE 2

COMET C/2002 S2 POSITION AND KinEMATIC PARAMETERS AT THE TIME OF UVCS COMET OBSERVATIONS

\begin{tabular}{|c|c|c|c|c|c|c|c|r|}
\hline $\begin{array}{c}t_{\text {enter }} \\
(\mathrm{UT})\end{array}$ & $N_{\exp }$ & $\begin{array}{c}r \\
\left(R_{\odot}\right)\end{array}$ & $\begin{array}{c}\rho_{\text {obs }} \\
\left(R_{\odot}\right)\end{array}$ & $\begin{array}{c}\alpha \\
(\mathrm{deg})\end{array}$ & $V_{r}^{\#}$ & $V_{\text {los }}^{\#}$ & $V_{\text {pos }}^{\#}$ & $\mathrm{~F}_{\alpha}{ }^{*}$ \\
\hline $21: 05: 21$ & 10 & 8.54 & 7.40 & 30 & 197 & 31 & -209 & 45 \\
$21: 32: 31$ & 24 & 8.02 & 6.84 & 31 & 202 & 35 & -215 & 192 \\
$22: 29: 39$ & 21 & 6.97 & 5.72 & 35 & 214 & 45 & -230 & 239 \\
$23: 18: 26$ & 28 & 5.99 & 4.66 & 39 & 227 & 57 & -246 & 474 \\
\hline
\end{tabular}

$\#\left(\mathrm{~km} \mathrm{~s}^{-1}\right)$

$*\left(\mathrm{ph} \mathrm{cm}^{-2} \mathrm{~s}^{-1}\right)$

The comet kinematic parameters are given by the velocity components toward the Sun, $V_{r}$, along the lineof-sight, $V_{\text {los }}$, and in the plane of the sky, $V_{\text {pos }}$. The comet light curve from LASCO C2 and $\mathrm{C} 3$ observations is shown in Figure 3. The apparent magnitude of the comet coma is plotted as a function of time, thus with decreasing heliocentric distance. The brightness differs somewhat from the values shown in Figure 2 of Knight et al. (2010) because we have not corrected the observed magnitudes for the phase angle, but both light curves show the dramatic minimum near $6 R_{\odot}$. We note that the coma brightness increases as the comet approaches the Sun and it reaches a peak at $\sim 12 R_{\odot}$ then it decreases up to $\sim 5.7 R_{\odot}$ at this point the brightness shows a sudden increase which continues until the comet is observed at the closest heliocentric distance of $\sim 3.0 R_{\odot}$. The increasing comet brightness above $12 R_{\odot}$ is a result of increasing solar flux and an increasing outgassing rate. After the peak the sublimation rate of the coma dust is higher than its production rate, giving the decreasing brightness observed for all the Kreutz sungrazing comets (Biesecker et al. 2002; Knight et al. 2010). Finally, the increase at lower distances might be related to fragmentation events at the distance of minimum of the brightness $\left(\sim 5.7 R_{\odot}\right)$ or farther from the Sun (Bemporad et al.
2005). The apparent H I Ly $\alpha$ magnitude from UVCS data, superposed on the visible magnitude from LASCO in Figure 3 is discussed in Section 2.2.

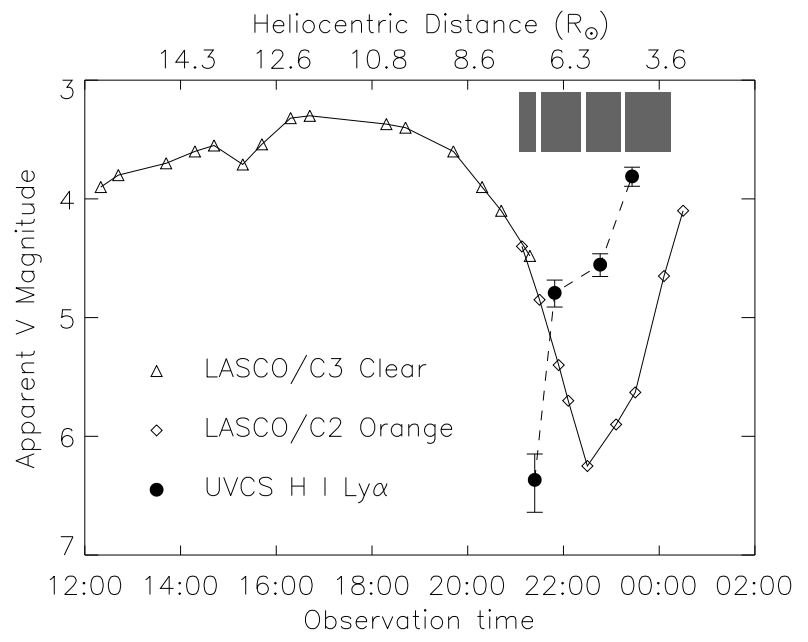

FIG. 3.- Apparent V magnitude of Comet C/2002 S2 determined from LASCO C2 and C3. The black dots represent the apparent magnitudes derived from the UVCS observations of the H I Lya line arbitrarily scaled to the LASCO apparent magnitude range.

\subsection{UVCS Observations and Spectral Data Analysis}

UVCS observed Comet C/2002 S2 on 2002 September, 18 between 20:36 UT and 00:15 UT of the next day. The instrument roll to catch the comet was $236^{\circ}$ counterclockwise from north pole and the heliocentric distance ranges from $8.10 R_{\odot}$ to $4.66 R_{\odot}$. After an initial 120 second exposure at $8.10 R_{\odot}$, where a faint signal from the comet was observed, the data were acquired at 4 lower heights: $7.40,6.84,5.72$, and $4.66 R_{\odot}$ in a series of $120 \mathrm{~s}$ exposures. The number of exposures, $N_{\text {exp }}$, at each height is given in Table 2. The observations were designed so the coverage at each height includes some exposures before and/or after the comet enters the UVCS slit to provide information about the coronal and interplanetary H I Ly $\alpha$ background. The UV spectral and spatial binning were $0.183 \AA$ ( 2 pixels) and $21^{\prime \prime}$ ( 3 pixels) respectively. The slit width was $150 \mu \mathrm{m}$, which corresponds to an integration region of $42^{\prime \prime}$ in the direction of the comet path. In the UV spectral range covered (975 to $1223 \AA$ to $1039.35 \AA$ ) the comet was detectable only in the $\mathrm{H} \mathrm{I} \mathrm{Ly} \alpha 1215.67 \AA$ line. The UVCS position angle, $236^{\circ}$ from solar north, was successfully chosen so that the comet crossed near the center of the slit and the comet path is perpendicular to the slit length, as shown also in Figure 2. The temporal sequence of $\mathrm{H} \mathrm{I}$ Ly $\alpha$ spectral images acquired when the comet crossed the lowest observed height, i.e. $\rho_{o b s}=4.66 R_{\odot}$, is reported in Figure 4 . The emission increases as the comet nucleus enters the slit (first three exposures), and then fades as the hydrogen is ionized away. We can see also the increasing size of the H I comet cloud due to the thermalization of the outgassed comet hydrogen with the coronal protons. The uncertainty in the determination of comet position with the UVCS data, besides the calibration uncertainties, is due to the slit width and the comet movement through the slit during the integration time with a ve- 
locity range from $\approx 209$ to $\approx 246 \mathrm{~km} \mathrm{~s}^{-1}$ at the different observed heights, therefore it is estimated to be at least $0.04 R_{\odot}$.
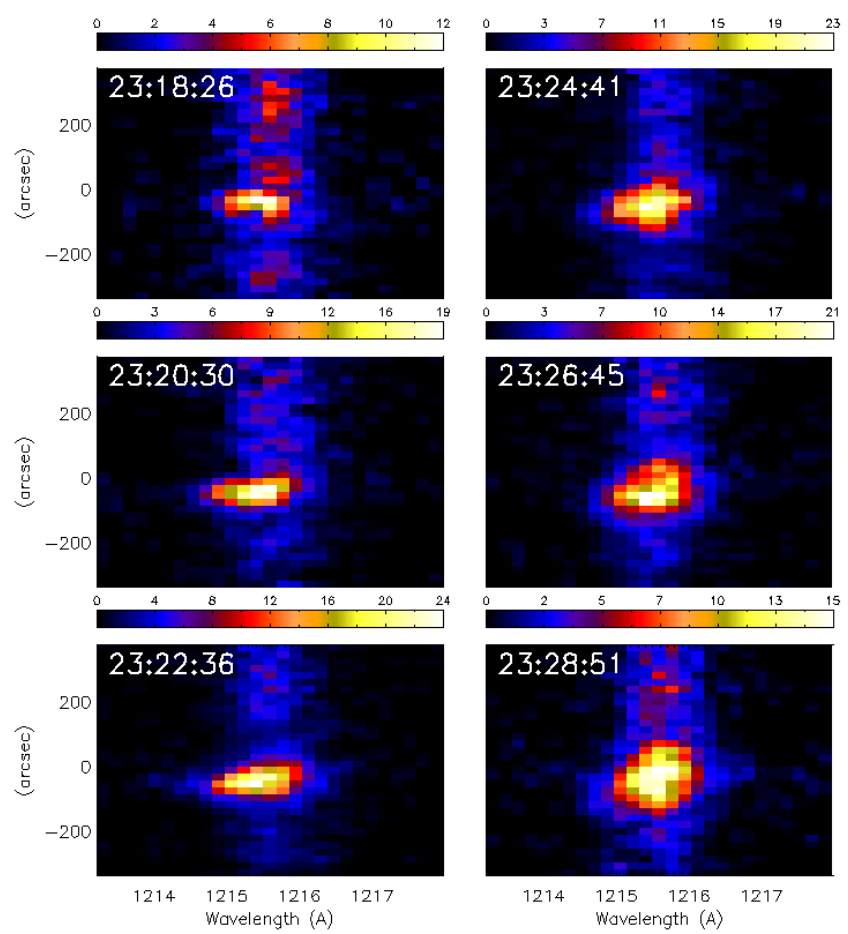

FIG. 4.- Sample temporal sequence of $\mathrm{H}$ I Ly $\alpha$ spectral images at the time of comet crossing UVCS slit at $\rho_{o b s}=4.66 R_{\odot}$. The color bars show the total count per pixel collected in 120 second exposures. In each panel the $\mathrm{x}$-axis represents the wavelength and the $\mathrm{y}$-axis the position along the slit.

Before the comet observation, the UVCS daily synoptic program, which lasted about 12 hours, was performed by scanning the $360^{\circ}$ corona at eight polar angles and several heliocentric heights. In order to remove possible instrumental effects in the comet spectra, we perform an evaluation of the spatial flat field along the slit, by combining all the $\mathrm{H}$ I Ly $\alpha$ counts in the spectral direction for all the synoptic scans performed on 2002 September, 18. Then we normalized the profile along the slit by a smoothed profile to obtain the flat field which is then applied to the spectral data. The analysis of spectral data is performed after the subtraction, at each height, of the off-line background, evaluated in a detector region where no lines are expected, and of the background from the exposures before the comet. In this way we remove the background emission due to coronal, stray light and interplanetary $\mathrm{H}$ I $\mathrm{Ly} \alpha$.

The background emission can be used to evaluate the coronal proton temperatures before the comet crossing. Stray light and interplanetary emission are very narrow with respect to the coronal line, so the background values have to be considered lower limits to the coronal proton temperatures. Thus, by taking into account the instrumental broadening, the coronal kinetic temperature can be estimated as $T_{k} \sim 1.1 \times 10^{6} \mathrm{~K}$ at $\rho_{\text {obs }}=4.66 R_{\odot}$ decreasing to $T_{k} \sim 6.4 \times 10^{5} \mathrm{~K}$ at $\rho_{\text {obs }}=6.84 R_{\odot}$; we note that these are average temperatures along the lines of sight, so they do not necessarily apply to the regions the comet crossed.
The H I Ly $\alpha$ comet light curves, that is the intensity as a function of time, are computed by integrating the spectra over \pm 300 " along the slit around the comet center and fitting them by a gaussian profile convolved with the instrumental profile. The light curves are shown in Figure 5 for the 4 observed heights. We see that, at the lowest height $\left(\rho_{o b s}=4.66 R_{\odot}\right.$, black curve) the emission increases quite rapidly and slowly fades to pre-comet values, while at higher distances the growth is more gradual. Finally, at the largest observed distance $\left(\rho_{\text {obs }}=7.40 R_{\odot}\right.$, green curve), the signal is very noisy and useless for spectral analysis. We point out that, at the time of the first contact, the real signal could be larger than observed because the comet is not yet completely into the UVCS slit.

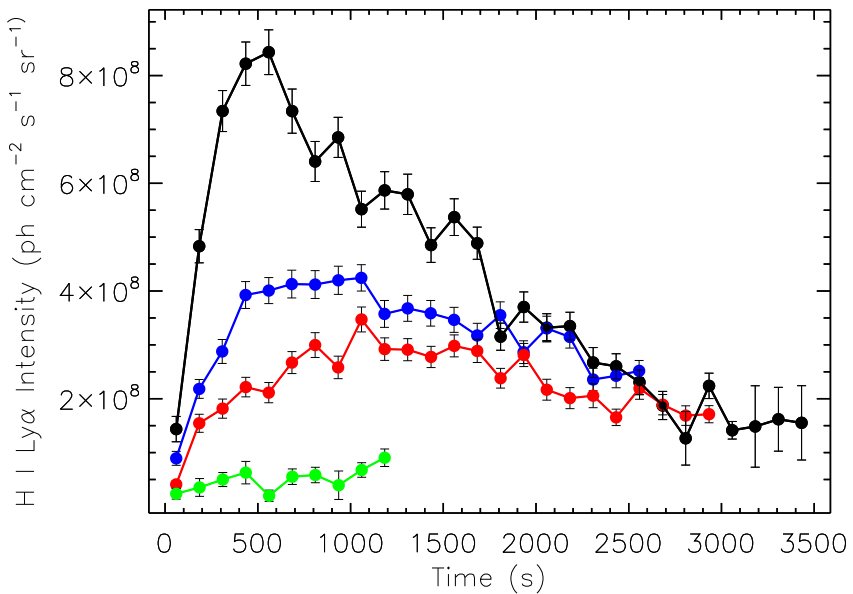

FIG. 5.- Observed H I Ly $\alpha$ intensity (photons $\mathrm{cm}^{-2} \mathrm{~s}^{-1} \mathrm{sr}^{-1}$ ) as a function of time integrated over a region of \pm 300 " centered on the comet axis from observations at $\rho_{\text {obs }}=4.66 R_{\odot}$ (black), $\rho_{\text {obs }}=5.72 R_{\odot}$ (blue), $\rho_{\text {obs }}=6.84 R_{\odot} \quad\left(\right.$ red) and $\rho_{\text {obs }}=7.40 R_{\odot}$ (green).

From gaussian fitting of the cometary spectra integrated along the slit we also determine the centroid of H I Ly $\alpha$ line with respect to the background corona. Whereas at the 3 higher heights the values are comparable, within the uncertainties, to the pre-corona emission, at $4.66 R_{\odot}$ a clear trend from a $100 \mathrm{~km} \mathrm{~s}^{-1}$ blue-shift toward the velocity of the background corona is found, as shown in the left panel of Figure 6 . We note that the spectrum in the first exposure when the comet is coming into the UVCS slit can be blue-shifted because the comet does not completely fill the slit, so the signal is coming primarily from a region close to the edge of the slit further from the Sun. Therefore, for the first comet exposure, the centroid shift is probably overestimated.

We also compare the line width of $\mathrm{H}$ I Ly $\alpha$ line with the values from background exposures. The comet velocity distribution is always comparable with the background. No trend is evident as a function of time, except for the first exposure at each height. The clearest difference from background is observed at $4.66 R_{\odot}$ (see right panel of Figure 6 ), here we point out that the narrow line width in the first exposure is due either to partial filling of the UVCS aperture or to the pre-charge transfer component of H I near the comet nucleus. The line width gives information about the $\mathrm{H}$ I Ly $\alpha$ photon production mechanism. On the one hand, if the signal comes from solar radiation scattered by $\mathrm{H}$ I atoms created by photo- 

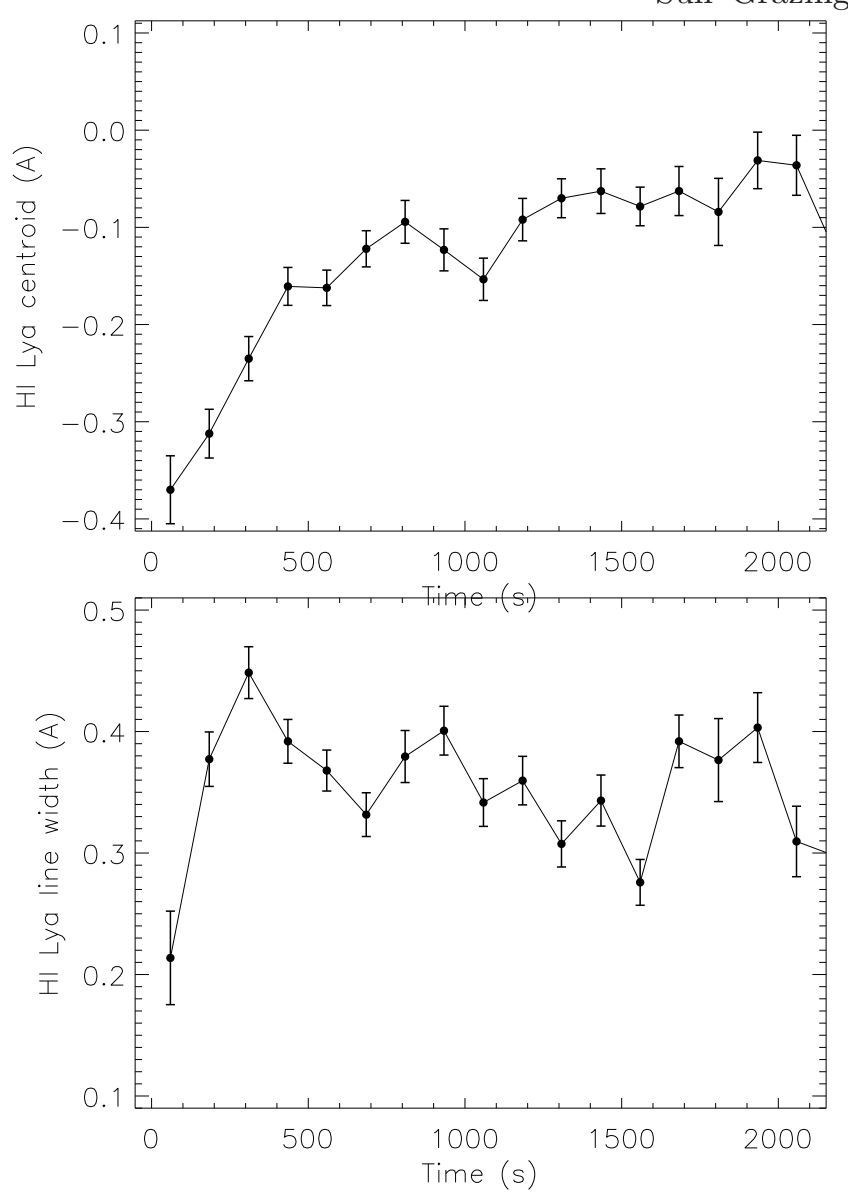

Fig. 6.- Observed H I Ly $\alpha$ centroid (upper panel) with respect to the background corona and $\mathrm{H}$ I Ly $\alpha$ line width (bottom panel) as a function of time integrated over a spatial region of \pm 300 " around the comet axis from observations at $\rho_{o b s}=4.66 R_{\odot}$

dissociation of water, we expect a narrow line profile because of the low speeds of those atoms. On the other hand, line widths close to that observed in the ambient corona are expected for photons scattered by $\mathrm{H}$ I atoms after charge transfer with coronal protons.

We reconstruct 2-dimensional comet images from UVCS spectral data using the known comet velocity in the plane of the sky, $V_{\text {pos }}$, based on LASCO observations and ephemeris computation. At each of the 4 observed heights the comet is detected as it crosses the UVCS slit at a fixed distance from the Sun. For each exposure the intensity of the $\mathrm{H}$ I $\mathrm{Ly} \alpha$ line at each position along the slit is measured. Then the exposures are shifted in the radial direction by $r_{\text {exp }}=V_{\text {pos }}\left(t_{\text {exp }}-t_{\text {enter }}\right)$ where $t_{\text {exp }}$ is the time of the beginning of the exposure and $t_{\text {enter }}$ is the time of the first comet observation at each height. Because the comet motion is perpendicular to the slit we do not need to shift the exposures in the direction parallel to the slit length.

The images of the UV comet emission from neutral hydrogen are shown in Figure 7. All the images are 600" wide centered on the radial axis at polar angle of $236^{\circ}$ counterclockwise from north pole, and the extent in the direction perpendicular to the comet path for the different heights depends on the comet velocity and the number of available exposures. The H I Ly $\alpha$ tail due to interaction of outgassed neutral hydrogen with the coro- nal protons, widens with time because of the random thermal motions of the second generation $\mathrm{H}$ I atoms.
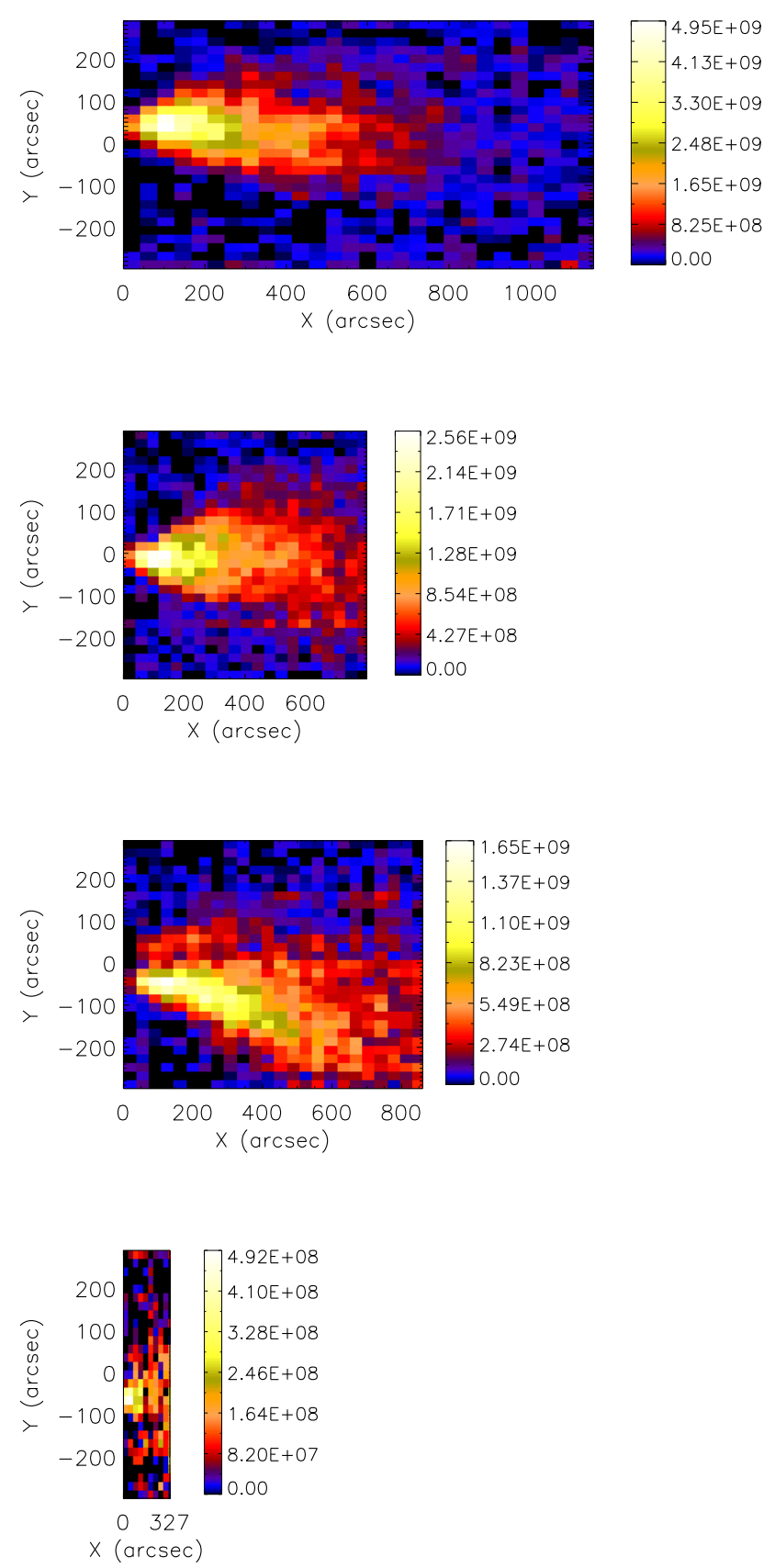

Fig. 7.- Comet H I Ly $\alpha$ intensity images from UVCS observations at $\rho_{o b s}=4.66 R_{\odot}, \rho_{o b s}=5.72 R_{\odot}, \rho_{o b s}=6.84 R_{\odot}$ and $\rho_{\text {obs }}=7.40 R_{\odot}$ from top to bottom.

The reconstructed 2-dimensional images obtained by the UVCS spectrometer can be compared to the images obtained in visible light by LASCO. We show the same portion of the sky observed from UVCS and LASCO C2 in Figures 8. We can see that from Sep 18 21:27 to 22:21 UT, UVCS clearly observes two comet tails at $6.84 R_{\odot}$, whereas a single, very narrow tail is imaged at $6.90 R_{\odot}$ by LASCO C2 in a 25 seconds exposure at 21:30 UT. The 
LASCO tail is presumably the dust tail, which is much brighter than the ion tail in other sungrazing comets (Ciaravella et al. 2010). The northern part of the UVCS image seems to correspond to the LASCO tail, whereas the southern part deviates from the comet trajectory.
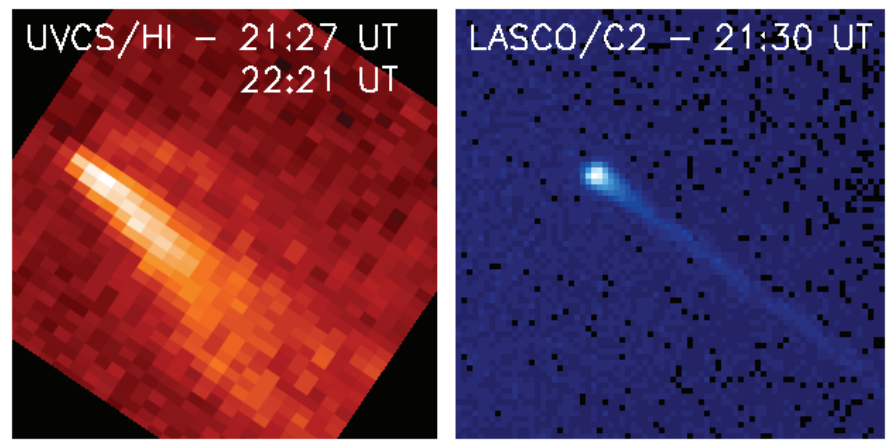

Fig. 8.- Comet image in H I Ly $\alpha$ from UVCS (left) and in white light from LASCO/C2 (right) for the same region of sky at approximately the same time. The size of the images is $855 \times 855$ ". The image pixel size is 11.4" for LASCO/C2 telescope, while for UVCS it is 21 arcsec along the slit and is $\sim 33$ " in the direction parallel to the comet path, the latter value is due to the comet movement during the exposure time.

At the three lower heights with a suitable spatial binning the signal-to-noise ratio is adequate to study the structure of the $\mathrm{H}$ I Ly $\alpha$ line centroid along the slit by fitting the spectral lines. Therefore, we reconstruct the 2D Doppler images of the comet, that is the line centroid deviation from the background values. As displayed in Figure 9, at all the heights, the upper part of the comet (closer to the equator) is blue-shifted with respect the background and the lower part shows a smaller, but clear red-shift, which is mainly evident at $6.84 R_{\odot}$. At all heights, the blue-shifts reach values $>100 \mathrm{~km} \mathrm{~s}^{-1}$, whereas the red-shifts reach about $80 \mathrm{~km} \mathrm{~s}^{-1}$. We discard the hypothesis of an instrumental effect because the profiles along the slit of the line centroid of the background exposures do not show any significant trend, and only the first exposures are expected blue-shifted for instrumental reasons, as shown in the left panel of Figure 6 .

\section{ANALYSIS}

Previous studies of sungrazers observed by UVCS (Uzzo et al. 2001; Bemporad et al. 2005) derived the parameters of the comet and of the solar wind based on a simple semi-analytic model which assumes that the outgassed H I atoms travel with the nucleus and that a single timescale describes the ionization and charge transfer processes. In this work, we compare the observations with a more detailed model based on Monte Carlo simulations. Before describing the simulations, we summarize the physical processes involved, the rates we assume, and the likely ranges of the parameters of the comet and coronal plasma.

\subsection{Physical Processes and Rates}

As a comet approaches the Sun, water is very quickly photodissociated into hydrogen and oxygen atoms through the reactions:

$$
\mathrm{H}_{2} \mathrm{O}+h \nu \rightarrow \mathrm{OH}+H
$$
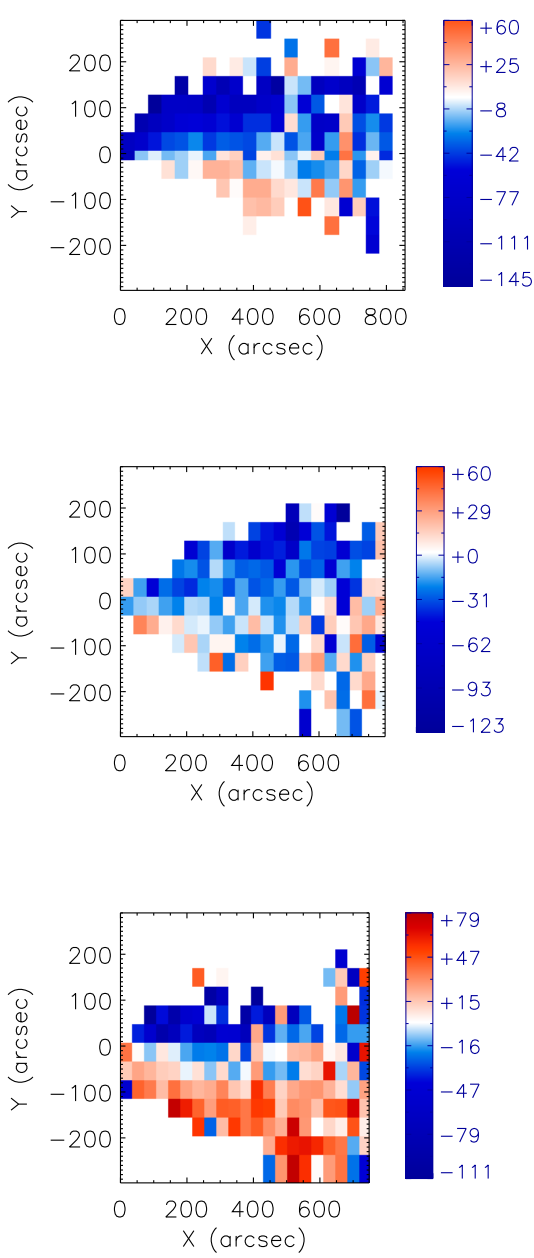

Fig. 9.- Doppler speed images of the H I Ly $\alpha$ comet emission from UVCS observations at $\rho_{\text {obs }}=4.66 R_{\odot}$ (upper), $\rho_{\text {obs }}=5.72 R_{\odot}$ (center) and $\rho_{\text {obs }}=6.84 R_{\odot}$ (bottom). The color bars show the Doppler speed in $\mathrm{km} \mathrm{s}^{-1}$.

$$
\mathrm{OH}+h \nu \rightarrow \mathrm{O}+H .
$$

creating a first generation of neutral $\mathrm{H}$ atoms with speeds of 8 (from $\mathrm{OH}$ ) to 20 (from $\mathrm{H}_{2} \mathrm{O}$ ) $\mathrm{km} \mathrm{s}^{-1}$ (Combi \& Smyth 1988; Mäkinen et al. 2001a) with respect to the nucleus. If the density is high enough, collisions between hydrogen atoms, molecules and heavier atoms will slow the $\mathrm{H}$ atoms to a few $\mathrm{km} \mathrm{s}^{-1}$. These $\mathrm{H}$ atoms can resonantly scatter chromospheric $\mathrm{H}$ I Ly $\alpha$ photons, but because they are more or less moving at the speed of the comet, the scattering is subject to strong Doppler dimming (Swings effect) at the comet radial speeds above the half width of the chromospheric emission profile, or about $100 \mathrm{~km} \mathrm{~s}^{-1}$ (Lemaire et al. 2002a). Noci \& Maccari (1999) give the cross section and angular dependence of this process. As discussed in greater detail below, the population of neutrals formed by photodissociation has a modest optical depth near the comet, which will reduce the number of scattered photons by $5 \%$ - $50 \%$ for the outgassing rates and solar wind densities considered here.

The first generation $\mathrm{H}$ atoms outgassed from cometary nucleus are subject to collisional ionization by electron 
impact and photoionization, which reduces the neutral atom population, but most will undergo charge transfer with coronal protons and generate a second generation population. At typical coronal electron temperatures $\left(T_{e} \approx 10^{6} \mathrm{~K}\right)$ and densities $\left(n_{e} \approx 10^{3}-10^{5} \mathrm{~cm}^{-3}\right)$, the collisional ionization rate is

$$
\tau_{\text {coll }}^{-1}=\gamma n_{e} \mathrm{~s}^{-1}
$$

where $\gamma \approx 2.78 \times 10^{-8} \mathrm{~cm}^{3} \mathrm{~s}^{-1}$ (Scholz \& Walters 1991), and the photoionization rate is

$$
\tau_{p h}^{-1} \approx 2 \times 8.3 \times 10^{-5}\left(\frac{6.8}{r}\right)^{2} \mathrm{~s}^{-1}
$$

as calculated by Ravmond et al. (1998) for a comet observation at solar minimum, where $r$ is the heliocentric distance in $R_{\odot}$. We have increased the photoionization rate by a factor of two to account for the increased UV emission near solar maximum. The charge transfer process between the first generation neutral $\mathrm{H}$ atoms and the solar wind protons produces a population of neutral hydrogen with nearly the same velocity distribution, kinetic temperature and bulk speed as the solar wind protons with a rate

$$
\tau_{e x}^{-1}=\left(\sigma_{e x} n_{e} v_{e f f}\right) \mathrm{s}^{-1}
$$

where $\sigma_{e x}=1.3 \times 10^{-15} \mathrm{~cm}^{2}$ (Schultz et al. 2008), is the charge transfer cross section and $v_{e f f}$, the effective velocity for charge transfer, is the sum (in quadrature) of the proton thermal speed, $V_{t h}$, and the comet speed relative to the solar wind, $V_{r e l}=V_{r}-V_{w}$, where $V_{w}$ is the solar wind speed. At the observed heights, charge transfer is the most rapid process. The post-charge transfer neutrals also scatter chromospheric $\mathrm{H}$ I $\mathrm{Ly} \alpha$ and the similarities between the cometary and coronal H I Ly $\alpha$ profiles indicate that these second generation $\mathrm{H}$ atoms are the main source of the $\mathrm{H} \mathrm{I}$ Ly $\alpha$ comet emission. Collisional excitation of $\mathrm{H}$ I $\mathrm{Ly} \alpha$ is generally less important than photoexcitation at the densities and heliocentric distances considered here, even when Doppler dimming is strong. From an observational point of view, the collisional excitation can be discarded by considering that the $\mathrm{H}$ I $\mathrm{Ly} \beta$ emission from this comet is negligible, as was found in previously observed sungrazers (Ravmond et al. 1998; Uzzo et al. 2001). The ratio of $\operatorname{Ly} \beta$ to $\operatorname{Ly} \alpha$ is about 0.001 for radiative excitation and 0.16 for collisional excitation, so the non-detection $\operatorname{Ly} \beta$ implies that the collisional contribution to $\operatorname{Ly} \alpha$ is small.

These processes, along with the neutral $\mathrm{H}$ outgassing rate, $\dot{N}_{H}=2 \dot{N}_{\mathrm{H}_{2} \mathrm{O}}$, and the coronal parameters, such as the electron density, $n_{e}$, and the solar wind outflow speed, $V_{w}$, determine the cometary H I Ly $\alpha$ light curve. The temperature of the corona also enters, in that the extent of the H I Ly $\alpha$ emission along the UVCS slit depends the speed of the second generation neutrals, and therefore on the temperature of the coronal protons from which they form. The coronal proton kinetic temperature in principle can be determined from observations of the background corona before the comet crosses the slit, but the values obtained are line-of-sight averages that may differ from the values at the actual position of the comet. In addition, the measured coronal spectra include narrow background and foreground interplanetary
H I Ly $\alpha$ and stray light from the solar disk not fully suppressed by the telescope occulters (Cranmer et al. 2010). For this reason, we simulated three different temperatures from 0.8 to $1.5 \times 10^{6} \mathrm{~K}$. Based on other sungrazing comets we expect $\dot{N}_{H} \sim 10^{28}$ to $10^{29}$ per second. The expected electron densities are in the range from current sheet to coronal hole values at solar maximum from Guhathakurta et al. (2006). We expect wind speeds of 50 to $250 \mathrm{~km} \mathrm{~s}^{-1}$ from the UVCS observations of Strachan et al. (2002) and the LASCO observations of Sheelev et al. (1997). Higher wind velocities would imply such strong Doppler dimming that the resonantly scattered $\operatorname{Ly} \alpha$ would be faint, but the $\operatorname{Ly} \alpha$ to $\operatorname{Ly} \beta$ ratio shows that this is not the case.

We neglect solar radiation pressure and gravity effects in the computation because they are very small for sungrazing comets with respect to the above processes. We also neglect the effect of the cometary material on the dynamics of the solar wind and the interaction with the magnetic field. We address the latter effect in the Discussion section.

\subsubsection{Optical Depth}

One important physical correction to the earlier analyses of UV observations of sungrazing comets (Ravmond et al. 1998; Uzzo et al. 2001; Bemporad et al. 2005) is the effect of finite optical depth of the H I Ly $\alpha$ line. For larger comets at greater distances from the Sun, detailed radiative transfer calculations have been performed by Combi \& Smyth (1988), and the optical depth has been inferred from the $\mathrm{H} \mathrm{I} \mathrm{Ly} \alpha$ intensity profile (e.g., Raymond et al. 2002) A cloud of hydrogen expanding at a speed V has a density $\dot{N}_{H} / 4 \pi r^{2} V$, and the H I Ly $\alpha$ scattering cross section is inversely proportional to the velocity width. Therefore, the opacity is proportional to $V^{-2}$ and optical depth effects are far more important for the first generation atoms $\left(V \sim 10 \mathrm{~km} \mathrm{~s}^{-1}\right)$ than for the second $\left(V>100 \mathrm{~km} \mathrm{~s}^{-1}\right)$.

To model the effects of opacity in the H I Ly $\alpha$ line, we assumed a spherical cloud with $\dot{N}_{H}$ particles per second injected at the center with an expansion velocity $\mathrm{V}$ and a radius $r_{c}$ at which the neutrals are destroyed by ionization or removed from the slowly expanding population by charge transfer. The radius of that cloud is $r_{c}=V \times \tau_{d}$, where $\tau_{d}=\left(1 / \tau_{p h}+1 / \tau_{\text {coll }}+1 / \tau_{c t}\right)^{-1}$ is the lifetime of neutral atoms. The density is

$$
n=\frac{\dot{N}_{H}}{4 \pi r^{2} V} e^{-r / r_{c}}
$$

The H I Ly $\alpha$ scattering cross section averaged over the line profile is

$$
\sigma=0.026 f \lambda / \Delta V \sim 1.3 \times 10^{-7} / \Delta V \mathrm{~cm}^{2}
$$

where $f$ is the oscillator strength, $\lambda$ is the wavelength of the transition and $\Delta V$ is the FHWM of $\mathrm{H} \mathrm{I}$ Ly $\alpha$ line.

For a grid of values of the coronal density, wind speed, and $\dot{N}_{H}$, we compute the suppression factor

$$
S_{\text {fact }}=\frac{\int n e^{-\tau}}{\int n}
$$

for density, $n$, and optical depth, $\tau=\sigma \times \int n$, averaged over the cloud. 
As mentioned above, the optical depth is important only for the first generation neutrals, and the expansion speed, hereafter called attenuation speed, $V_{a t t}$, is a critical parameter. Hydrogen atoms are formed at speeds of 8 to $20 \mathrm{~km} \mathrm{~s}^{-1}$ by photo-dissociation of $\mathrm{H}_{2} \mathrm{O}$ and $\mathrm{OH}$. If the dissociation occurs where the density is low, they expand with that range of speeds. However, much of the photodissociation can occur where the density is high enough that the gas is collisional, especially at the high temperatures expected close to the Sun. For the observation at $4.66 R_{\odot}$ the suppression factor of $\mathrm{H}$ I Ly $\alpha$ for the pre-charge transfer component has been computed for grids of models with $V_{a t t}=16.0,5.0,3.5 \mathrm{~km} \mathrm{~s}^{-1}$, in the $\dot{N}_{H}$ range from $10^{27}$ to $10^{30} \mathrm{~s}^{-1}, V_{w}$ from 50 to 250 $\mathrm{km} \mathrm{s}^{-1}$ and coronal density from $1 \times 10^{3}$ to $5 \times 10^{4} \mathrm{~cm}^{-3}$. For example, with $\dot{N}_{H}=2 \times 10^{28} \mathrm{~s}^{-1}, V_{w}=100 \mathrm{~km} \mathrm{~s}^{-1}$ and $n_{e}=1.75 \times 10^{3} \mathrm{~cm}^{-3}$ the suppression factors are $0.71,0.25$ and 0.15 for $V_{a t t}=16,5$ and $3.5 \mathrm{~km} \mathrm{~s}^{-1}$, respectively. This suppression factor is applied to scattering from all first generation atoms, while second generation atoms are assumed to be in the optically thin regime in the Monte Carlo simulations.

\section{MONTE CARLO SIMULATION}

In order to better understand the relationships between the observed H I Ly $\alpha$ spectral emission and the physical parameters of the coronal plasma encountered by the comet we developed a simulation code based on the Monte Carlo technique. The purpose is to reproduce the observed sungrazer H I Ly $\alpha$ spectra, and therefore the reconstructed comet light curves and images, as functions of coronal and cometary parameters.

At each observed height we run a large number of simulations for a grid of coronal and cometary input parameters. The coronal parameters are the electron density, $n_{e}$, the wind velocity, $V_{w}$ and the proton kinetic temperature, $T_{k}$. We choose different values, $n_{e, 0}$ and $V_{w, 0}$, at the comet observed radial distance, $r_{0}$, then we define the electron density radial profile as given by Guhathakurta et al. (2006) and the wind velocity radial profile from mass flux conservation. The kinetic temperature is assumed constant in the radial range of interest. The only cometary grid parameter is the neutral hydrogen outgassing rate at the comet observed distance, $\dot{N}_{0}$, which is assumed to decrease inversely proportional to square distance: $\dot{N}_{H}(r)=\dot{N}_{0}\left(\frac{r_{0}}{r}\right)^{2}$.

A simulation run begins with a generation of a sample of neutral hydrogen atoms at a heliocentric distance far from the UVCS field of view, moving with the bulk velocity of the comet plus a spherically symmetric outgassing velocity distributed in the range 8 to $20 \mathrm{~km} \mathrm{~s}^{-1}$ (Combi \& Smyth 1988; Mäkinen et al. 2001b). We start the simulations far enough from the UVCS slit that less than $1 \%$ of the number of particles generated at the first time step reaches the UVCS field of view. The comet position and its kinematic parameters evolve as functions of time following the trajectory computed from orbital parameters (see Tables 1 and 2). At each simulation time step, $\delta t=5 \mathrm{~s}$, a number of new particles ( $\mathrm{H}$ atoms) proportional to $\dot{N}_{H}(r)$ is generated at the new position, $r$, and added to the previous particles. Then the simulated atoms can be subject to two different processes; ionization or charge transfer, as discussed in Section 3.1. Based on the position of each particle and the coronal electron density, we compute the collisional, $\tau_{\text {coll }}^{-1}$, and photoionization $\tau_{p h}^{-1}$, rates (Equations 3 and 4 ), and the ionization probability for each particle in the $\delta t$ time interval is $P_{\text {ion }}=1-\exp \left[-\left(\delta t / \tau_{\text {ion }}\right)\right]$, where $\tau_{\text {ion }}^{-1}=\tau_{\text {coll }}^{-1}+\tau_{p h}^{-1}$. With the Monte Carlo method we statistically remove the ionized atoms, reducing their number. Similarly, the charge transfer probability is driven by the rate given in Equation 5, where we need to account for the comet, wind and coronal thermal velocities at the position of each particle. All the particles that undergo charge transfer get new velocities given by the composition of the radial wind velocity and the random thermal speed. At each time step we update the position $\vec{P}(x, y, z)$ and velocity $\vec{V}\left(v_{x}, v_{y}, v_{z}\right)$ of all particles in the three-dimensional space, where $x$ and $y$ defines the plane of the sky and $z$ the line-of-sight direction.

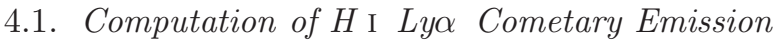

The only relevant mechanism to produce $\mathrm{H}$ I Ly $\alpha$ emission is resonant scattering of the chromospheric radiation. This process is characterized by the so-called g-factor (e.g., Oxenius 1965; Mäkinen et al. 2001b), the number of photons per second scattered by each atom, which for Ly $\alpha$ resonance of neutral hydrogen at $1 \mathrm{AU}$ can be expressed as

$$
g_{A U}=\frac{\pi e^{2}}{m_{e} c} f_{\alpha} \frac{\lambda_{0}^{2}}{c} F_{\odot}
$$

where $f_{\alpha}$ is the $\mathrm{H}$ I $\operatorname{Ly} \alpha$ oscillator strength, $\lambda_{0}$ is the reference wavelength in $n m, F_{\odot}$ is exciting specific solar flux in $p h \mathrm{~cm}^{-2} \mathrm{~s}^{-1} \mathrm{~nm}^{-1}, e$ and $m_{e}$ are the electron charge and mass. We scale the previous equation to the heliocentric distance, $r$ measured in $R_{\odot}$ units, of each simulated scattering particle by the dilution factor, $\Omega_{r} / \Omega_{A U}$, that is the ratio between the solid angles subtended by the source of resonant radiation at $1 \mathrm{AU}$ and at the distance $r$. Following Noci et al. (1987), we compute the solid angles as $\Omega=2 \pi\left(1-\sqrt{1-\frac{1}{r^{2}}}\right)$, and we take into account the angular dependence of the H I Ly $\alpha$ scattering through the function $p(\phi)=\frac{11+3 \cos ^{2} \phi}{12}$, where $\phi$ is the scattering angle between the Sun-particle vector and the line-of-sight. Therefore, for each simulated particle we compute the number of scattered photons with:

$$
g=\frac{\Omega_{r}}{\Omega_{A U}} \frac{11+3 \cos ^{2} \phi}{12} \frac{\pi e^{2}}{m_{e} c} f_{\alpha} \frac{\lambda_{0}^{2}}{c} F_{\odot}
$$

where the H I Ly $\alpha$ exciting specific flux, $F_{\odot}$, measured by SUMER/SOHO (Lemaire et al. 2002b), is selected as the value corresponding to the radial velocity, $v_{r}$, of each scattering particle. Finally, for each scattering particle we determine the emitted wavelength, $\lambda$, from the line-of-sight velocity, $v_{z}$, of the particle itself as $\lambda=\lambda_{0}\left(1-\frac{v_{z}}{c}\right)$, where $\lambda_{0}$ is the rest wavelength of the H I Ly $\alpha$ transition.

From the number of photons scattered per second by each simulated particle we compute the emitted spectra by combining, along the line- of-sight (los), all the photons from all the atoms in each simulated spatial element defined as the UVCS spatial resolution. The emission 
per unit of wavelength is obtained by collecting all the photons emitted in each spectral window with resolution $\Delta \lambda=0.09 \AA$. In this way, for each spatial element in the plane of the sky $(\Delta x \Delta y)$, we determine the H I Ly $\alpha$ specific intensity, $I(\lambda)$ in $p h \mathrm{~cm}^{-2} s^{-1} s r^{-1} A^{-1}$ as

$$
I(\lambda)=\frac{1}{4 \pi} \frac{1}{\Delta x \Delta y} \frac{1}{\Delta \lambda} \sum_{\text {los }} g
$$

where the factor $1 / 4 \pi$ normalizes the emission to unit of solid angle and $1 / \Delta \lambda$ to unit of wavelength. Because the simulated time steps are shorter than the UVCS exposure time, we average the intensity from all the time steps over the UVCS integration time of a single exposure. Finally, the simulated spectra are multiplied by the ratio between the assumed outgassing rate, $\dot{N}_{0}$, and the number of simulated outgassed particles per second. In this way we obtain intensity spectra directly comparable to the radiometric calibrated spectra from UVCS observations.

\subsection{Model Grid of Parameters}

Table 3 summarizes the grid of the free parameters used to simulate the comet observation at the lowest height, $\rho_{o b s}=4.66 R_{\odot}$, which corresponds to an actual heliocentric distance $\mathrm{r}=5.99 R_{\odot}$. The outgassing rate, at the comet observed distance, assumes values from $10^{27}$ to $10^{30} \mathrm{~s}^{-1}$ as expected from previous UVCS sungrazing comet observations (Ravmond et al. 1998; Uzzo et al. 2001; Bemporad et al. 2005; Ciaravella et al. 2010) We ran simulations for wind velocities from 50 to $200 \mathrm{~km} \mathrm{~s}^{-1}$, with $25 \mathrm{~km} \mathrm{~s}^{-1}$ steps and also with $V_{w, 0}=0 \mathrm{~km} \mathrm{~s}^{-1}$ as a control value which is not physically significant, and with $V_{w, 0}=250 \mathrm{~km} \mathrm{~s}^{-1}$, which ultimately develops a very faint signal because of Doppler dimming. The electron density can assume ten different values from 0.05 to 1.92 times the radial profile determined by Guhathakurta et al. (2006) for coronal current sheets, and three different values $\left(8 \times 10^{5}, 1.1 \times 10^{6}\right.$ and $1.5 \times 10^{6} \mathrm{~K}$ ) for coronal kinetic temperature are modeled. We ran a model neglecting the optical depth and three models with different attenuation speeds (3.5, 5.0 and $16.0 \mathrm{~km} \mathrm{~s}^{-1}$, see section 3.1 .1 ), giving different suppression factors for the emission from pre--charge transfer atoms.

TABLE 3

Simulation GRID FREE PARAmEters AT $\rho_{o b s}=4.66 R_{\odot}$

\begin{tabular}{|l|l|l|c|}
\hline Quantity & Description & Range & Steps \\
\hline$\dot{N}_{0}$ & Outgassing rate & $10^{27}$ to $10^{30}$ atoms s & 61 \\
$V_{w, 0}$ & Wind velocity & 0 to $250 \mathrm{~km} \mathrm{~s}^{-1}$ & 9 \\
$n_{e, 0}$ & Electron density & $1.1 \times 10^{3}$ to $4.1 \times 10^{4} \mathrm{~cm}^{-3}$ & 10 \\
$T_{k}$ & Proton temperature & $0.8 \times 10^{6}$ to $1.5 \times 10^{6} \mathrm{~K}$ & 3 \\
$V_{a t t}$ & Attenuation speed & 0 to $16 \mathrm{~km} \mathrm{~s}^{-1}$ & 4 \\
\hline
\end{tabular}

\subsection{Simulation-Observation Comparison}

For all the possible combinations of these free parameters we simulate the H I Ly $\alpha$ spectra emitted by the comet crossing the UVCS slit field of view. By integrating the spectra over the wavelength and over the spatial direction along the slit we obtain the total intensity as a function of time as the comet crosses the UVCS slit, that is the simulated light curves comparable with the those observed (see Figure 5). Then we compare the observations with each simulation by computing the $\chi_{\nu}^{2}$ defined as

$$
\chi_{\nu}^{2}=\frac{1}{\nu} \sum_{N} \frac{\left(I_{o b s}-I_{s i m}\right)^{2}}{\sigma_{o b s}^{2}+\sigma_{s i m}^{2}}
$$

where $N$ is the number of data points, $\nu=N-n-1$ is the number of degrees of freedom with $n=5$ is the number of parameters of the model, $I_{o b s}$ and $I_{\text {sim }}$ are the observed and simulated intensities and $\sigma_{o b s}$ and $\sigma_{\text {sim }}$ their statistical standard deviations. In this way we obtain a multi-dimensional array $\chi_{\nu}^{2}\left(\dot{N}_{0}, V_{w, 0}, n_{e, 0}, T_{k}, V_{a t t}\right)$, which quantitatively summarizes the comparison of all simulated models with observations. Then minimizing this parameter determines the set of coronal and cometary parameters which best fit the observations.

First of all, we search for the best fit for the four different corrections of the opacity (see Table 3). We found that the models with the intermediate speed values, $V_{a t t}=3.5$ and $5.0 \mathrm{~km} \mathrm{~s}^{-1}$, produce comparable results, both matching the observed data at $4.66 R_{\odot}$ quite well (see blue and red models in Figure 10), whereas the models which neglect the opacity and those with higher speed show spikes of narrow, red-shifted emission for 1 or 2 exposures when the comet enters the slit which are not seen in the data (Figure 10). Therefore, we choose the $V_{a t t}=3.5 \mathrm{~km} \mathrm{~s}^{-1}$ model, which suppresses spike for all but the most extreme choices of other parameters. We point out that the approximation of two hydrogen atom populations, from water $\left(20 \mathrm{~km} \mathrm{~s}^{-1}\right)$ and from $\mathrm{OH}\left(8 \mathrm{~km} \mathrm{~s}^{-1}\right)$ photodissociation, should work well for larger heliocentric distances $(>0.8 \mathrm{AU}$ ) Combi et al. 2005), whereas at small heliocentric distances the velocity distribution emerging from the collision zone is expected to pile- $\mathrm{up}$ at low velocities $\left(<4 \mathrm{~km} \mathrm{~s}^{-1}\right)$ (e.g., Combi \& Smyth 1988).

The best fit between the observed and simulated comet light curves at $\rho_{o b s}=4.66 R_{\odot}$, corresponding to the radial distance $r=5.99 R_{\odot}$ is obtained with the following coronal parameters; $V_{w, 0}=75 \mathrm{~km} \mathrm{~s}^{-1}, n_{e, 0}=1.23 \times 10^{4} \mathrm{~cm}^{-3}$ and $T_{k}=8 \times 10^{5} \mathrm{~K}$ and with the comet outgassing rate $\dot{N}_{0}=1.12 \times 10^{28} s^{-1}$.

To verify that the best fit corresponds to a minimum of the $\chi_{\nu}^{2}$ distribution, we show the two-dimensional maps of $\chi_{\nu}^{2}$ for selected values of three parameters. For example with fixed $T_{k}=8.0 \times 10^{5} \mathrm{~K}$ and $V_{\text {att }}=3.5 \mathrm{~km} \mathrm{~s}^{-1}$ we show the $\chi_{\nu}^{2}\left(\dot{N}_{0}, V_{w, 0}\right)$ map with $n_{e, 0}=1.23 \times 10^{4} \mathrm{~cm}^{-3}$ (top panel of Figure 11), the $\chi_{\nu}^{2}\left(\dot{N}_{0}, n_{e, 0}\right)$ map with $V_{w, 0}=75 \mathrm{~km} \mathrm{~s}^{-1}$ (middle panel) and the $\chi_{\nu}^{2}\left(V_{w, 0}, n_{e, 0}\right)$ map with $\dot{N}_{0}=1.12 \times 10^{28} \mathrm{~S}^{-1}$ (bottom panel). As shown in the maps, the $\chi_{\nu}^{2}$ distribution has a minimum around the values of the parameters which define the best fit.

From the simulation runs that best fit the light curves we reconstruct the expected $\mathrm{H}$ I Ly $\alpha$ intensity $2 \mathrm{D}$ images with the same procedure used to build the images of Figure 7. The simulated images are shown in Figure 12. The images match quite well the observed data except that the comet nucleus is too bright in the simulation due to spatial blurring of the observational data which effectively reduces the spatial resolution. 


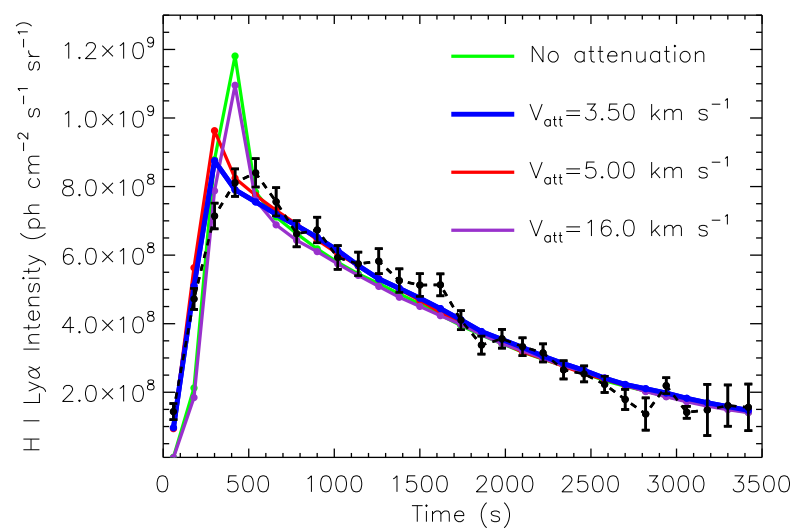

FIG. 10.- Observed and best-fit comet light curve at $\rho_{\text {obs }}=4.66 R_{\odot}$ with different optical depth assumptions. Black dots with error bars are the observed H I Ly $\alpha$ intensity. The green curve represents the best simulated light curve without correction for optical depth. The blue curve represents the correction with the attenuation speed of $3.5 \mathrm{~km} \mathrm{~s}^{-1}$, the red curve with $5.0 \mathrm{~km} \mathrm{~s}^{-1}$ and the violet one with $16 \mathrm{~km} \mathrm{~s}^{-1}$.
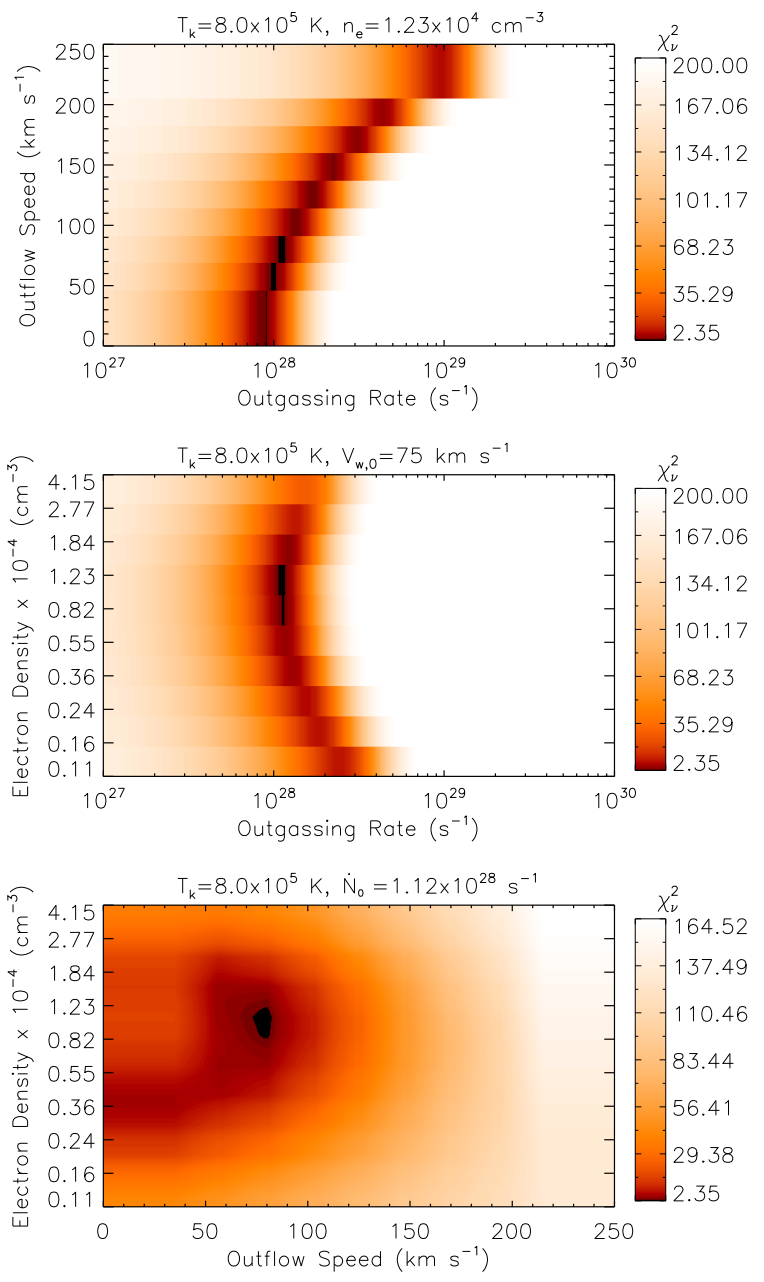

FIG. 11.- Example of $\chi_{\nu}^{2}$ maps. Top: $\chi_{\nu}^{2}\left(\dot{N}_{0}, V_{w, 0}\right)$, middle: $\chi_{\nu}^{2}\left(\dot{N}_{0}, n_{e, 0}\right)$, bottom: $\chi_{\nu}^{2}\left(V_{w, 0}, n_{e, 0}\right)$ 
As a further check on the goodness of fit we compute the variation of the size of the tail in the direction perpendicular to the comet path, that is along the UVCS slit, and compare it with the observations. The tail size is defined as the $1 / e$ distance from the intensity peak determined from a gaussian fit of the total intensity profile along the slit. Figure 13 shows that the best fit model for the light curve also provides a good description of the tail size observed at $\rho_{o b s}=4.66 R_{\odot}$.

The gaussian fitting of simulated spectra also provides the variation of the line centroid and line width as the comet crosses the instrument slit. We find that for the first two exposures, the simulated $\mathrm{H}$ I Ly $\alpha$ line is red-shifted by $\approx+0.28 \AA$ and the line is very narrow $(\approx 0.12 \AA$ ) because the signal comes from the cold first generation atoms moving with the nucleus away from the observer. The line width is in line with the observations (right panel of Figure 6), but the line centroid values are puzzling. In first several exposures, there are large blue-shifts that cannot be due to instrumental effects (see also Section 2.2), whereas the simulation predicts spectral lines with small blue-shifts, $\approx 25-30 \mathrm{~km} \mathrm{~s}^{-1}$, as expected from ions moving with a $75 \mathrm{~km} \mathrm{~s}^{-1}$ solar wind at $39^{\circ}$ to the line-of-sight. That is in line with the 0.1 to $0.2 \AA$ shifts seen in most of the exposures.

We ran simulations for higher heights $\left(\rho_{\text {obs }}=5.72 R_{\odot}\right.$ and $6.84 R_{\odot}$ ) with similar grids of parameters, except that the electron density grids are centered at the expected values at those heights. The best fit between the observed and simulated light curves at $\rho_{\text {obs }}=5.72 R_{\odot}$ is shown in Figure 13. The coronal parameters are $V_{w, 0}=75$ $\mathrm{km} \mathrm{s}^{-1}, n_{e, 0}=7.73 \times 10^{3} \mathrm{~cm}^{-3}$ and $T_{k}=8 \times 10^{5} \mathrm{~K}$ and the outgassing rate is $\dot{N}_{0}=8.91 \times 10^{27} \mathrm{~s}^{-1}$. As for the previous height, the best fit is obtained with an attenuation velocity of $3.5 \mathrm{~km} \mathrm{~s}^{-1}$.

As we can see in the observed light curves at larger heights (Figure 5), the $\mathrm{H} \mathrm{I} \mathrm{Ly} \alpha$ signal takes up to about $1000 \mathrm{~s}$ to reach the emission peak, then it slowly decreases. This trend is difficult to reproduce with our model which predicts a rapid rise when the comet nucleus reaches the slit, somewhat smoothed by the opacity and instrumental effects, followed by a rapid fading as it leaves the slit and a gradual fading after that. A possible explanation is the presence of many fragments traveling along the comet path, as supported by the LASCO C2 images of the comet archived with the Planetary Data System (Knight 2008). White light images have been processed by subtracting the median of four neighboring images to remove the background. Figure 15 shows the images at the time of UVCS observation at $5.72 R_{\odot}$. We can see the clear difference between the two images taken with a time interval of 36 minutes: in the second one the nucleus is not well localized and the comet path seems to be a collection of many bright points. However, this explanation seems at odds with the rapid rise seen at the lowest height.

Another explanation for the the slow fading of $\mathrm{H}$ i Lya might be a tail of pyroxene grains. Kimura et al (2002) suggested that such grains could account for both the secondary peak in LASCO brightness inside $7 R_{\odot}$ and also for the increased Ly $\alpha$ emission at small heights (Uzzo et al. 2001) if pyroxene sublimates and acts to neutralize protons in the corona. Bemporad et al 2005 attributed a very slowly decaying $\operatorname{Ly} \alpha$ signal in comet $\mathrm{C} / 2001 \mathrm{C} 2$ to sublimation of dust grains in the comet tail and subsequent charge transfer interactions. A 0.1 $\mu \mathrm{m}$ pyroxene grain is expected to survive for about 1000 seconds at about $5 R_{\odot}$ before it sublimates (Kimura et al 2002).

None of the models could match the light curve at the third height $\left(\rho_{\text {obs }}=6.84 R_{\odot}\right.$; see Figure 16$)$. One problem is that the relatively low speed of the comet at this height leads to a bright spike due to the first generation neutrals. The second problem is the very slow rise. We discuss model limitations that could cause these problems, along with the red- and blue-shifts seen at different positions along the slit at lower heights, in the next section.

\section{DISCUSSION}

The Monte Carlo model allows us to interpret UV and optical light curves in terms of the coronal and cometary parameters and the ion and dust contributions. A more complete model will be needed to analyze the interaction between the ions produced by the comet and the ambient solar wind and coronal magnetic field, but we can make an initial assessment, discuss and summarize our results.

\subsection{Coronal density, temperature and solar wind}

At the two lower observed heights, the model can reproduce the $\mathrm{H}$ I Ly $\alpha$ comet light curves and the size of the comet tail perpendicular to comet path. Table 4 gives the results of the model fits; we point out that the determined parameters are related to the actual heliocentric distance, $r$, whereas $\rho_{o b s}$ is just the projection on the plane of the sky. The coronal electron densities are compared with the values derived for the solar maximum coronal current sheet and coronal hole by Guhathakurta et al. (2006), which can be taken as the lower and upper limits to coronal density (Figure 17). The uncertainty on the density is given mainly by the grid of values used for the simulation. The best fit wind velocity is $75 \mathrm{~km} \mathrm{~s}^{-1}$ at both heights, though at $\mathrm{r}=6.97 R_{\odot}$ a value of $100 \mathrm{~km} \mathrm{~s}^{-1}$ is also acceptable, as expected from the wind acceleration. Higher wind velocities do not predict persistent $\mathrm{H}$ I comet tails because of Doppler dimming. One way to get acceptable light curves with higher wind speeds would be to simulate a number of well separated fragments, but that is beyond the scope of this paper. The proton kinetic temperature is typically $8 \times 10^{5} \mathrm{~K}$, as confirmed by the size of the comet tail in the direction perpendicular to its path and from the line widths.

TABLE 4

Best model parameters

\begin{tabular}{|c|c|c|c|c|c|c|}
\hline $\begin{array}{c}r \\
R_{\odot}\end{array}$ & $\begin{array}{c}\rho_{\text {obs }} \\
R_{\odot}\end{array}$ & $\begin{array}{c}n_{e, 0} \\
\mathrm{~cm}^{-3}\end{array}$ & $\begin{array}{c}V_{w, 0} \\
\mathrm{~km} \mathrm{~s}^{-1}\end{array}$ & $\begin{array}{l}T_{k} \\
\mathrm{~K}\end{array}$ & $\begin{array}{c}N_{0} \\
\mathrm{~s}^{-1}\end{array}$ & $\begin{array}{c}V_{\text {att }} \\
\mathrm{km} \mathrm{s}^{-1}\end{array}$ \\
\hline 5.99 & 4.66 & $1.23 \times 10^{4}$ & 75 & $8.0 \times 10^{5}$ & $1.12 \times 10^{28}$ & 3.5 \\
\hline 6.97 & 5.72 & $7.73 \times 10^{3}$ & 75 & $8.0 \times 10^{5}$ & $8.91 \times 10^{27}$ & 3.5 \\
\hline
\end{tabular}

\subsection{Outgassing rates and size of nucleus}

As the comet approaches the Sun, it experiences a rapid increase in illumination, and most of the energy of the solar radiation goes into the sublimation of water. 

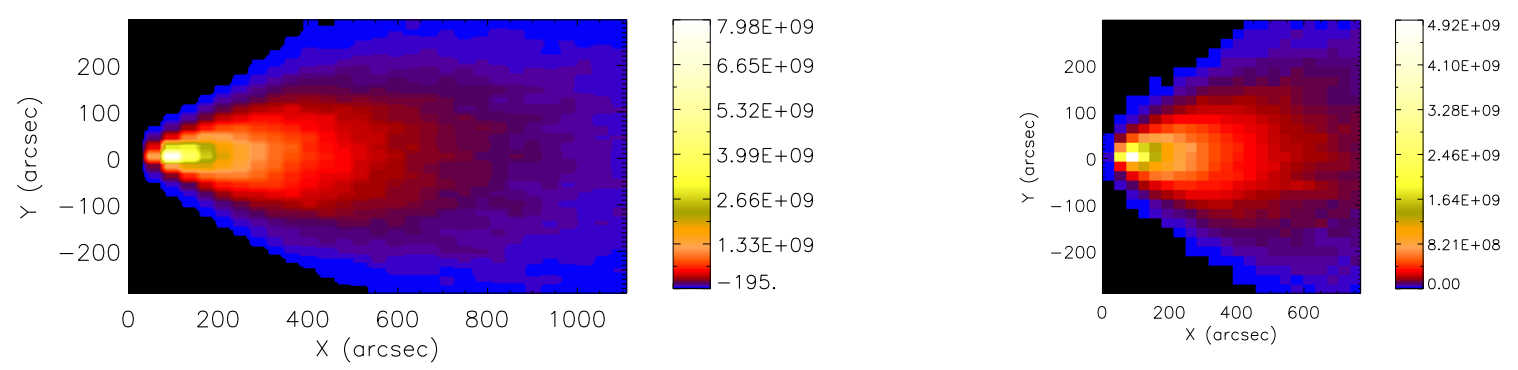

FIG. 12. - H I, Ly $\alpha$, intensity images reconstructed from the best simulation at $\rho_{\text {obs }}=4.66 R_{\odot} \quad\left(\right.$ left panel) and at $\rho_{\text {obs }}=5.72 R_{\odot}($ right panel)

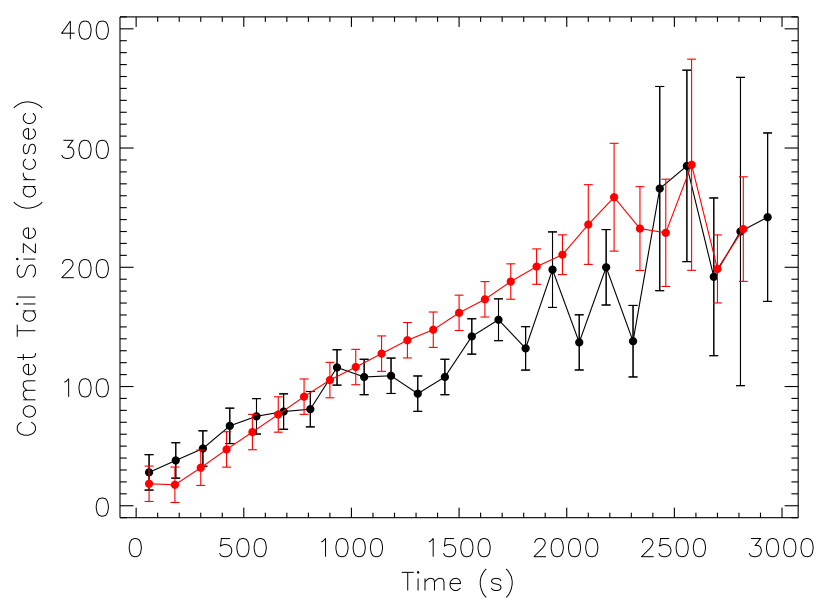

FIG. 13. - Simulated (red) and observed (black) comet tail size at $\rho_{\text {obs }}=4.66 R_{\odot}$.

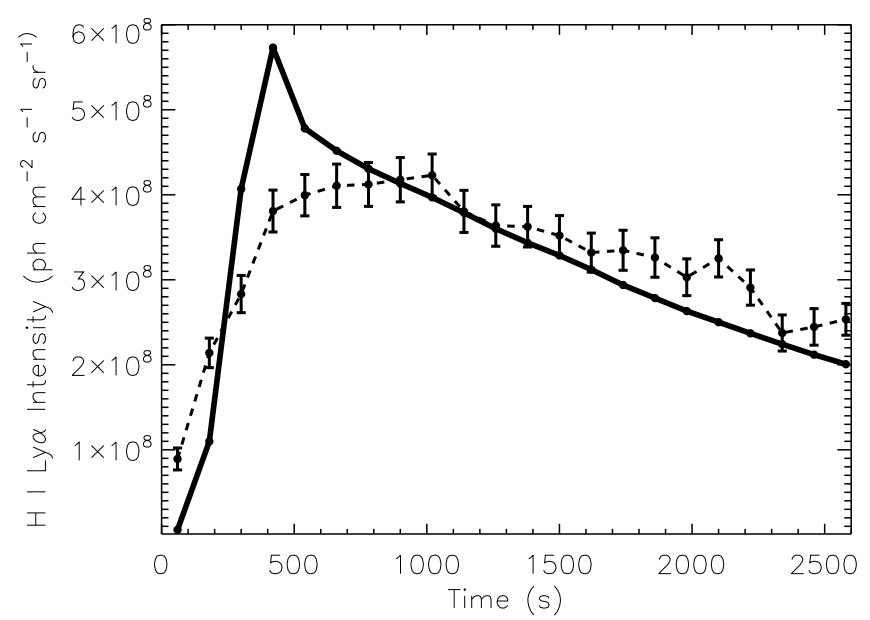

FIG. 14. - Best fitting of simulated (black) and observed (black dots with error bar) comet light curve at $\rho_{\text {obs }}=5.72 R_{\odot}$ with the attenuation velocity of $3.5 \mathrm{~km} \mathrm{~s}^{-1}$ 
The expected water outgassing rate, $\dot{N}_{\mathrm{H}_{2} \mathrm{O}}$ in molecules per second, can be estimated by assuming a balance between the energy supplied by the solar radiation over the cometary active surface, $S_{a c t}$, and the energy required to sublimate the quantity of ice corresponding to $\dot{N}_{\mathrm{H}_{2} \mathrm{O}}$. Therefore, the energy balance can be written as

$$
F_{h} S_{a c t} \approx \dot{N}_{\mathrm{H}_{2} \mathrm{O}} \frac{L}{N_{A}} \frac{1}{1-A}
$$

where $F_{h}=F_{\odot} / h^{2}$ is the solar flux, in $\operatorname{erg} \mathrm{cm}^{-2} \mathrm{~s}^{-1}$, scaled to the cometary heliocentric distance in AU, $h=$ $\left(r / 215 R_{\odot}\right), F_{\odot}=1.37 \cdot 10^{6} \mathrm{erg} \mathrm{cm}^{-2} \mathrm{~s}^{-1}$ is the solar flux at $1 \mathrm{AU}, L=4.81 \cdot 10^{11} \mathrm{erg} \mathrm{mol}^{-1}$ is the latent heat sublimation of ice, $N_{A}=6.022 \times 10^{23}$ molecules mol $^{-1}$ is Avogadro's number and $A=0.06$ is the cometary albedo. In a spherical approximation, the active surface exposed to the solar radiation is $S_{a c t}=\pi r_{c o m}^{2}$, where $r_{c o m}$ is the equivalent cometary nucleus radius. From Equation 12, $\dot{N}_{\mathrm{H}_{2} \mathrm{O}}$ can be estimated as

$$
\dot{N}_{\mathrm{H}_{2} \mathrm{O}} \approx \pi F_{\odot} \frac{r_{c o m}^{2}}{h^{2}} N_{A} \frac{1-A}{L}
$$

The best fit models at the two lower heights provide estimates of the neutral hydrogen outgassing rate. By assuming that all the observed $\mathrm{H}$ originates in the dissociation of water we can determine water production rate as $Q_{\mathrm{H}_{2} \mathrm{O}}=18 \cdot \dot{N}_{\mathrm{H}_{2} \mathrm{O}} \cdot U$, where $\dot{N}_{\mathrm{H}_{2} \mathrm{O}}=\dot{N}_{H} / 2$ and $U=1.66 \times 10^{-27} \mathrm{~kg}$ is the Atomic Mass Unit. The values are reported in Table 5, with the collection of published results obtained at different heliocentric distances from sungrazers observed by UVCS.

In a spherical approximation, from the values of the water production rate, $\dot{N}_{\mathrm{H}_{2} \mathrm{O}}$, by using Equation 13, we can derive an estimate of the comet equivalent radius, $r_{\text {com }}$, as:

$$
r_{\text {com }}=h \sqrt{\frac{1}{\pi} \frac{\dot{N}_{\mathrm{H}_{2} \mathrm{O}} L}{F_{\odot}(1-A) N_{A}}}
$$

where all the terms are described in Section 3.1. Comet Lovejoy (C/2011 W3) was by far the largest sungrazing comet observed in recent years, with a diameter of about $400 \mathrm{~m}$ (McCauley et al. 2013).

If the comet has fragmented, as suggested by Figure 15, the inferred radii shown in Table 5 are upper limits. The sizes of the nucleus shown Table 5 do not show any increase in the effective diameter between the crossing at $6.97 R_{\odot}$ and the crossing at $5.99 R_{\odot}$ as seen between $5.88 R_{\odot}$ and $4.68 R_{\odot}$ for comet $\mathrm{C} / 2000 \mathrm{C} 6$ and interpreted as an indication of fragmentation by Uzzo et al. (2001). However, if the nucleus were really a $9.4 \mathrm{~m} \mathrm{di}-$ ameter sphere at $6.97 R_{\odot}$, it would shrink by about $1 \mathrm{~m}$ at the outgassing rate shown in Table 5 by the time it reached $5.99 R_{\odot}$. The smaller change in apparent diameter might suggest some partial fragmentation, but the inferred diameters are uncertain at at least the $10 \%$ level even in the absence of fragmentation.

a. Ravmond et al. (1998)

b. Uzzo et al. (2001)

c. Bemporad et al. (2005)

d. Ciaravella et al. (2010)

e. Main fragment
TABLE 5

Previously Published SUNGRAZING OUTGASSing RATES.

\begin{tabular}{|l|c|c|c|c|}
\hline $\begin{array}{l}\text { Comet } \\
\text { Name }\end{array}$ & $\begin{array}{c}\mathrm{r} \\
{\left[R_{\odot}\right]}\end{array}$ & $\begin{array}{c}\dot{N}_{H} \\
{\left[10^{28} \mathrm{~s}^{-1}\right]}\end{array}$ & $\begin{array}{c}Q_{\mathrm{H}_{2} \mathrm{O}} \\
{\left[\mathrm{kg} \mathrm{s}^{-1}\right]}\end{array}$ & $\begin{array}{c}r_{\text {com }} \\
{[\mathrm{m}]}\end{array}$ \\
\hline $\mathrm{C} / 2002 \mathrm{~S} 2$ & 5.99 & 1.12 & 167 & 9.0 \\
& 6.97 & 0.89 & 133 & 9.4 \\
$\mathrm{C} / 1996 \mathrm{Y}^{a}$ & 6.80 & 0.13 & 20.0 & 3.4 \\
$\mathrm{C} / 2000 \mathrm{C}^{b}$ & 3.88 & 0.71 & 71.8 & 3.0 \\
& 4.68 & 1.35 & 140.0 & 5.7 \\
& 5.88 & 0.33 & 34.6 & 3.4 \\
& 6.47 & 0.13 & 10.5 & 2.5 \\
$\mathrm{C} / 2001 \mathrm{C}^{c}$ & $4.98^{e}$ & 0.59 & 58.9 & 7.8 \\
& $4.98^{f}$ & 0.29 & 28.5 & 5.4 \\
$\mathrm{C} / 2003 \mathrm{~K}^{d}$ & 3.60 & 8.20 & 820 & 20.3 \\
& 3.37 & $40-170$ & $6000-25000$ & $29-60$ \\
\hline
\end{tabular}

f. Subfragment

\subsection{UV and Optical light curves}

The optical fluxes measured by LASCO include both solar continuum scattered by dust grains and resonantly scattered emissions, prominently Na I Knight et al. 2010). The sudden drop in brightness inside $11 R_{\odot}$ with no counterpart in $\mathrm{H} \mathrm{I} \mathrm{Ly} \alpha$ (Figure 3) indicates that it corresponds to a sudden change in the dust destruction rate, for instance sublimation of the olivine grains when their temperature exceeds about $1500 \mathrm{~K}$ (Kimura et al. 2002). The sudden drop also implies a change in the radiation due to $\mathrm{Na} \mathrm{I}$ and other atomic or molecular species. It may be that the temperature in the coma becomes high enough to dissociate the molecules and ionize $\mathrm{Na}$ I. Knight et al. (2010) find an $r^{-4}$ brightening inside $24 R_{\odot}$, which is attributed to the $r^{-2}$ dilution factor and $r^{-2}$ outgassing rate.

The optical and UV light curves in Figure 3 are strikingly different. The rapid increase in Ly $\alpha$ brightness shows that the outgassing rate is rising rapidly even as the optical brightness drops by an order of magnitude. Sungrazing comets typically brighten as $r^{-3.8}$ in the optical as they move from $24 R_{\odot}$ to $16 R_{\odot}$, reach a peak near $12 R_{\odot}$ (Biesecker et al. 2002; Knight et al. 2010) and fade by two or three magnitudes as they move in to around $6 R_{\odot}$. Some brighten again when they reach about 5 to $7 R_{\odot}, \mathrm{C} / 2002 \mathrm{~S} 2$ being an extreme case. The rapid fading inside $12 R_{\odot}$ is usually attributed to sublimation of dust grains (Biesecker et al. 2002; Uzzo et al. 2001; Kimura et al. 2002; Bemporad et al. 2005; Knight et al. 2010) and the brightening at smaller radii is attributed either to an increase in the outgassing rate as the comet nucleus fragments and exposes more surface area to sunlight (Biesecker et al. 2002; Uzzo et al. 2001) or to crystalline pyroxenes, a more refractory form of dust (Kimura et al. 2002) . The dust is probably composed primarily of silicates rather than carbonaceous compound (Kimura et al. 2002). Ciaravella et al. (2010) found a Si:C ratio of about 10 in the sublimated dust of another Kreutz sungrazer, and carbonaceous grains would rapidly vaporize at the temperatures expected this close to the Sun.

The optical fluxes measured by LASCO include both solar continuum scattered by dust grains and resonantly scattered features such as $\mathrm{Na}$ I as indicated by a difference of up to 1.5 magnitudes between observations 

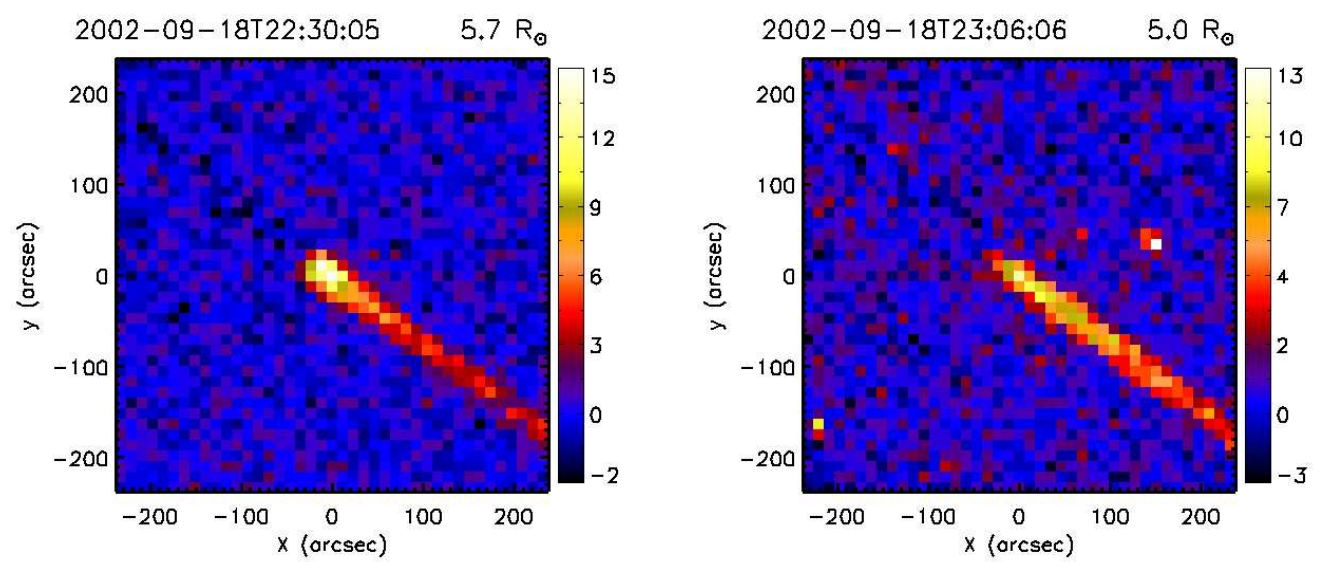

FIG. 15. - LASCO C2 white light comet images at the time of UVCS observation at $\rho_{\text {obs }}=5.72 R_{\odot}$, at 22:30UT (left panel) and at 23:06UT (right panel)

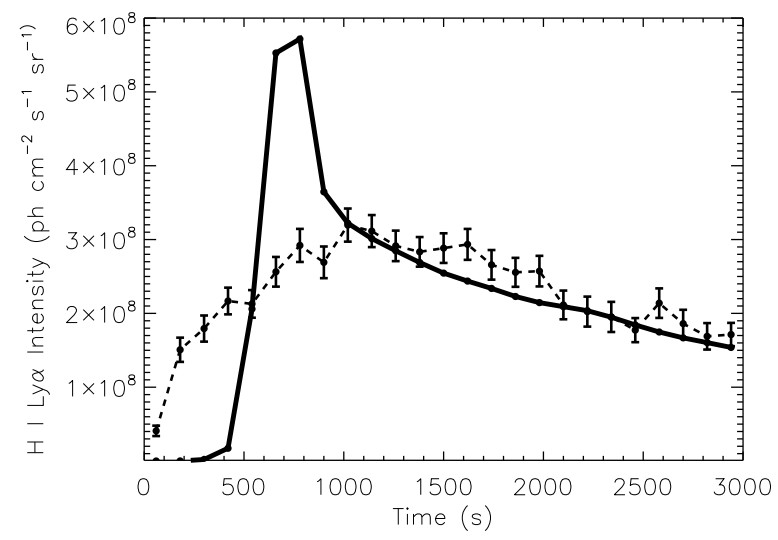

FIG. 16. - Best tail fitting of simulated (black) and observed (black dots with error bar) comet light curve at $\rho_{\text {obs }}=6.84 R_{\odot}$.

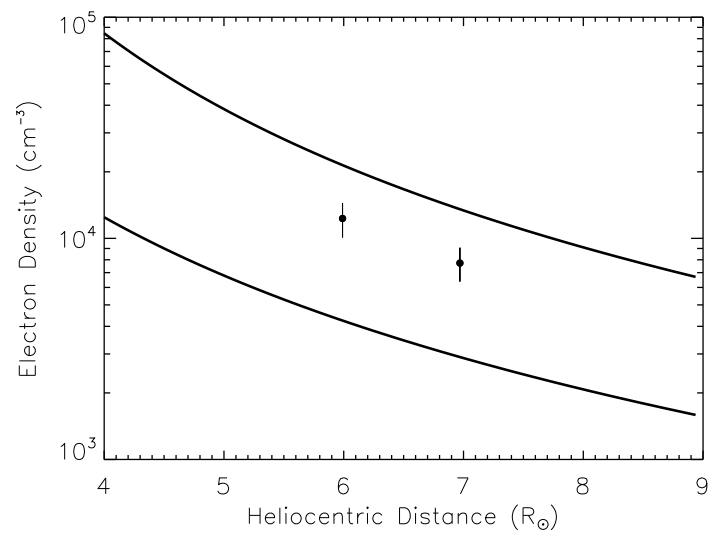

FIG. 17.- Coronal electron density at the heliocentric distance of UVCS comet observation (full dots with error bars). The two curves shows the electron density for current sheet and coronal hole at solar maximum measured by Guhathakurta et al. (2006) 
with the orange and clear filters (Knight et al. 2010; Lamy et al. 2013). The sudden drop in brightness suggests a change in the radiation due to $\mathrm{Na} \mathrm{I}$ and other atomic or molecular species as well as destruction of dust grains. It may be that the temperature in the coma becomes high enough to dissociate the molecules and ionize $\mathrm{Na}$ I. Biesecker et al. (2002) found that the emission line contribution is small by the time a sungrazer reaches 7 $R_{\odot}$, while Figure 2 of Knight et al. (2010), which shows C2 magnitudes for this comet systematically brighter than C3, suggests that the line contribution could be up to $30 \%$ of the $\mathrm{C} 2$ brightness.

\subsection{Disturbance of the corona by the comet}

Thus far we have treated the comet as a test particle, in that it has no effect of the solar wind it encounters. However, the comet will significantly perturb the wind in ways that can affect both the light curves and the Doppler shifts. As discussed by Galeev et al. (1985) and Gombosi et al. (1996), material from the comet mass loads the solar wind, and if the outgassing rate is large enough it can create a bow shock. The wind speed obtained by fitting the light curve should be the speed of the wind that has been slowed by the interaction.

We can estimate the importance of the dynamical effects of the interaction in two ways. First, we can estimate the size of the bow shock, and second we can compare the mass lost from the comet with the coronal mass in the interaction region.

The standoff distance, $r_{s t}$, from the comet nucleus is obtained by equating the ram pressure of the outflowing cometary gas to the ram pressure of the gas it encounters,

$$
\frac{\dot{N}_{H}}{4 \pi r_{\text {st }}^{2} V_{\text {out }}} \mu_{\text {com }} V_{\text {out }}^{2}=n_{\text {cor }} \mu_{\text {cor }} V_{\text {rel }}^{2}
$$

where $V_{\text {rel }} \approx V_{r}-V_{w}$ is the comet speed relative to the solar wind assumed to be radial, $\mu_{c o r}=1.8 \times 10^{-24} \mathrm{~g}$ is the coronal mass per $\mathrm{H}$ nucleus for a wind with $5 \%$ helium, and $\mu_{\text {com }}=1.49 \times 10^{-23} \mathrm{~g}$ is the mass per $\mathrm{H}$ nucleus for water. At the lowest observed comet height, $\mathrm{r}=5.99 R_{\odot}$, where the comet velocity is $V_{r}=227 \mathrm{~km} \mathrm{~s}^{-1}$, the standoff distance can be evaluated for the coronal and cometary parameters in Table 4 . For a coronal density $n_{e}=1.2 \times 10^{4} \mathrm{~cm}^{-3}$, a wind speed $V_{w} \approx 100 \mathrm{~km} \mathrm{~s}^{-1}$ an outgassing rate $\dot{N}_{H}=1.1 \times 10^{28} \mathrm{~s}^{-1}$ and an outgassing speed $V_{\text {out }} \approx 3.5 \mathrm{~km} \mathrm{~s}^{-1}$ from the optical depth analysis, the standoff distance is only $140 \mathrm{~km}$. The lateral extent of the bow shock size around twice this size, but even so, it is modest compared to the mean free path of the neutrals. Gombosi et al. (1996) presented a detailed model of a comet bow shock driven by outgassing hydrogen. They show that many of the neutrals charge exchange outside the bow shock, so that the mass-loaded flow slows down over a large region ahead of the shock. For the parameters relevant here, we expect a Mach number of around 3 , so the compression and heating will be modest.

A second estimate of the importance of the interaction is to compare the mass lost from the comet with the mass of the solar wind in the interaction volume. The comet mass loss rate is $\dot{N}_{H} \mu_{\text {com }}$. The outgassed atoms interact with the ambient corona through the lifetime before ionization, $\tau_{d}$, or about $950 \mathrm{~s}$ for a density of $1.2 \times 10^{4} \mathrm{~cm}^{-3}$ and a relative speed of $V_{\text {rel }} \sim 330 \mathrm{~km} \mathrm{~s}^{-1}$. The corresponding interaction volume is a cylinder whose length is $V_{r e l} \times \tau_{d}$, or $3.3 \times 10^{5} \mathrm{~km}$, and radius is the distance a particle travels during the interaction time, $V_{\exp } \times \tau_{d}$, where $V_{\text {exp }}$ is the expansion speed given by the rate of growth of the width of the tail seen in Figure 13. For $V_{\text {exp }}=75 \mathrm{~km} \mathrm{~s}^{-1}$ the cylinder radius is about 72,000 $\mathrm{km}$. Then the coronal mass in the interaction cylinder is about $10^{7} \mathrm{~kg}$, compared with an outgassed mass of about $4.8 \times 10^{5} \mathrm{~kg}$ in the time $\tau_{e x}$ for $\dot{N}_{H}=1.1 \times 10^{28} \mathrm{~s}^{-1}$. The actual size of the interaction region is smaller, because the oxygen atoms that account for most of the mass are moving more slowly, and no single scale length applies to all the interactions. Thus the comet significantly perturbs the solar wind with which it interacts, but does not dominate the flow. The sense of this perturbation is mostly a motion along the comet's path, which in this case is toward the Sun and away from the Earth.

One important effect of the disturbance in the solar wind velocity is that the bow shock created by the cometary plasma produces a region of heated coronal gas in the stagnation region at the tip of the bow shock which moves with the comet. Atoms that undergo charge transfer in that region produce a cloud of neutrals centered on the comet that expands at approximately the relative speed of the comet and the wind. Charge transfer in the flanks of the bow shock and in the region outside the bow shock that is compressed and accelerated by the interaction (Gombosi et al. 1996) will produce neutrals expanding more slowly about centroid velocities intermediate between the comet speed and the wind speed. Some of the neutrals produced in these heated, accelerated regions can move upstream faster than the comet. They account for the somewhat gradual rise of the light curve at $6.97 R_{\odot}$ and the even slower rise at $8.02 R_{\odot}$.

In addition, neutrals created near the stagnation region near the tip of the bow shock have a velocity centroid equal to that of the comet, so in the case of C/2002 S2 they are moving away from the Earth and might account for the red-shifts seen on the lower sides of the velocity images in Figure 9. However, it is not obvious why the red-shifted material would lie along one side of the comet trajectory, because to first order the bow shock is symmetric. The symmetry is broken by the fact that the comet speed is not antiparallel to the solar wind speed. In addition, the magnetic field can distort the bow shock in the same way that it affects the shape of the heliospheric termination shock (Lallement et al. 2005; Opher et al. 2009). A detailed model would be needed to determine whether this distortion could produce spatially separated red- and blue-shifted regions.

\subsection{Pickup Ion Behavior}

The pattern of blue-shifted emission to the north and red-shifted emission to the south seen in Figure 9 is quite remarkable. It has not been seen in other comets observed by UVCS, but that could be due to the viewing geometry or the lower signal-to-noise ratio of most of the other observations.

At first glance, the blue-shift can be explained as the line-of-sight component of the solar wind speed. For observed blue-shifts up to $100 \mathrm{~km} \mathrm{~s}^{-1}$ and the phase angles listed in Table 2, the solar wind speed would be of 
order 150 to $200 \mathrm{~km} \mathrm{~s}^{-1}$ if the wind is flowing radially. Those speeds agree reasonably well with streamer velocities at those heights (Strachan et al. 2002; Frazin et al. 2003; Abbo et al. 2010). However, as discussed in connection with the Monte Carlo models, speeds that large imply severe Doppler dimming and require correspondingly large outgassing rates that would be hard to reconcile with the lack of $\operatorname{Ly} \beta$ emission and the lack of a narrow spike from first generation hydrogen atoms. Models that match the light curves reasonably well predict blueshifts below about $60 \mathrm{~km} \mathrm{~s}^{-1}$.

The red-shift and its southward displacement must be related to the line-of-sight component of the comet's velocity away from the Earth. However, in detail the red-shift and displacement are complex to model. The most likely explanation for the red-shift along one edge of the comet's H I Ly $\alpha$ tail is related to the interaction of the cometary $\mathrm{H}$ atoms with the solar magnetic field. When a neutral moving with the comet is ionized in the coronal magnetic field, it behaves as a pickup ion, with its velocity component parallel to the field giving a flow along the field, whereas the perpendicular component becomes the gyro velocity around the field. The resulting ring beam in velocity space is unstable, and it rapidly evolves to a more isotropic bispherical shell in velocity space (Williams \& Zank 1994).

The resulting ions move along the field, as is seen in the striations observed in oxygen ions in Comet Lovejoy C/2011 W3 (Downs et al. 2013; Raymond et al. 2014). Because Comet $\mathrm{C} / 2002 \mathrm{~S} 2$ is moving away from the Earth, $V_{\|}$gives a red-shift. As seen in Comet Lovejoy, the gas can move to either side of the comet trajectory depending on the orientation of the magnetic field. Based on the observed red-shifts and the dominance of red-shift to one side of the comet tail, the line-of-sight component of the parallel speed is of order $80 \mathrm{~km} \mathrm{~s}^{-1}$, whereas the plane- of-the-sky component must be comparable to the thermal speed that causes the tail to expand, or $100 \mathrm{~km} \mathrm{~s}^{-1}$. In order for these pickup ions to become visible in $\operatorname{Ly} \alpha$, some of them must undergo a second charge transfer event to produce a population of neutrals with the pickup ion velocity distribution (third generation neutrals). The relatively high outgassing rate of Comet C/2002 S2 makes this second charge transfer more likely than in the smaller sungrazers observed by UVCS. If the plane-of-the-sky component of the solar wind speed tends toward the north, while the plane-ofthe-sky component $V_{\|}$tends toward the south, this explains the separation of red- and blue-shifted emission seen in Figure 9.

In earlier studies of sungrazing comets with UVCS, we were able to estimate the coronal density and temperature from the $\mathrm{H}$ I Ly $\alpha$ decay time and line width. The blue-shift adds a diagnostic for the solar wind speed, and the red-shift and the separation of the red- and blueshifted components offer a means to assess the magnetic field direction in 3 dimensions. However, the modeling needed to extract that information is a step beyond the models presented here.

\section{SUMMARY}

We report the UVCS/SOHO and LASCO/SOHO observations of sungrazing Comet C/2002 S2 at three heights, and we describe Monte Carlo simulations used to interpret the data in terms of the comet outgassing rate and to probe the coronal density, temperature and flow speed along its path. The Monte Carlo simulations are able to match the light curves and the expansion of the emission region along the UVCS slit at the lower two heights, but they do not match the light curve at the upper height. This comet is unlike others observed by UVCS in that it shows distinct red- and blue-shifts below and above the center of the images, and the Monte Carlo simulations do not predict such behavior.

There are two effects that were not included in the Monte Carlo models because they can be neglected for comets with small outgassing rates, but that are probably important for Comet C/2002 S2 and may account for the discrepancies between models and observations. First, the mass loading of the solar wind by cometary material slows the flow in the comet's frame of reference, compresses it and may cause a bow shock. Second, the protons produced when cometary neutrals are ionized by charge transfer, collisions with electrons or photoionization become pickup ions, and if those ions charge transfer with other neutrals they create a third generation population of neutrals with a distinctive mean velocity and velocity width. We plan to include the first effect in the next generation of Monte Carlo models. The second effect will be deferred to the more distant future because it is more complex, and it involves additional free parameters.

Because of the good capability of the Monte Carlo model to describe the H I Ly $\alpha$ emission from small sungrazers at low heliocentric distances, we plan to apply the method to other comets detected by UVCS, moreover we will adapt the code to compute the expected UV images, for a grid of coronal and cometary parameters, which shall be obtained by the coronagraphs aboard future space mission, such as the Solar Orbiter.

We are indebted to Brian Marsden, who performed the orbit calculations that made these observations possible. SG and JR thank the International Space Science Institute (Bern, Switzerland) for the opportunity to discuss this work within the International Study Team programme. $\mathrm{SOHO}$ is a project of international cooperation between ESA and NASA. UVCS is a joint project of NASA, Italian Space Agency (ASI), and the Swiss Funding Agencies. LASCO was built by a consortium of the Naval Research Laboratory, USA, the Laboratoire d'Astrophysique de Marseille (formerly Laboratoire d'Astronomie Spatiale), France, the Max-Planck-Institut für Sonnensystemforschung (formerly Max Planck Institute für Aeronomie), Germany, and the School of Physics and Astronomy, University of Birmingham, UK. This work was supported by NASA grant NAG5-12814.

Facilities: SOHO (UVCS, LASCO)

\section{REFERENCES}

Abbo, L., Antonucci, E., Mikić, Z., Linker, J. A., Riley, P., \& Lionello, R. 2010, Advances in Space Research, 46, 1400
Bemporad, A., Poletto, G., Raymond, J. C., Biesecker, D. A., Marsden, B., Lamy, P., Ko, Y., \& Uzzo, M. 2005, ApJ, 620, 523 
Biesecker, D. A., Lamy, P., St. Cyr, O. C., Llebaria, A., \& Howard, R. A. 2002, Icarus, 157, 323

Brueckner, G. E., Howard, R. A., Koomen, M. J., Korendyke, C. M., Michels, D. J., Moses, J. D., Socker, D. G., Dere, K. P., Lamy, P. L., Llebaria, A., Bout, M. V., Schwenn, R., Simnett, G. M., Bedford, D. K., \& Eyles, C. J. 1995, Sol. Phys., 162, 357

Ciaravella, A., Raymond, J. C., \& Giordano, S. 2010, ApJ, 713, L69

Combi, M. R., Mäkinen, J. T. T., Bertaux, J.-L., \& Quemérais, E. 2005, Icarus, 177,228

Combi, M. R. \& Smyth, W. H. 1988, ApJ, 327, 1044

Cranmer, S. R., Gardner, L. D., \& Kohl, J. L. 2010, Sol. Phys., 263, 275

Downs, C., Linker, J. A., Mikić, Z., Riley, P., Schrijver, C. J., \& Saint-Hilaire, P. 2013, Science, 340, 1196

Frazin, R. A., Cranmer, S. R., \& Kohl, J. L. 2003, ApJ, 597, 1145

Galeev, A. A., Cravens, T. E., \& Gombosi, T. I. 1985, ApJ, 289, 807

Gombosi, T. I., De Zeeuw, D. L., Häberli, R. M., \& Powell, K. G. 1996, J. Geophys. Res., 101, 15233

Guhathakurta, M., Sittler, E. C., \& Ofman, L. 2006, Journal of Geophysical Research (Space Physics), 111, 11215

Kimura, H., Mann, I., Biesecker, D. A., \& Jessberger, E. K. 2002, Icarus, 159, 529

Knight, M. M., A'Hearn, M. F., Biesecker, D. A., Faury, G., Hamilton, D. P., Lamy, P., \& Llebaria, A. 2010, AJ, 139, 926

Lallement, R., Quémerais, E., Bertaux, J. L., Ferron, S., Koutroumpa, D., \& Pellinen, R. 2005, Science, 307, 1447

Lamy, P., Faury, G., Llebaria, A., Knight, M. M., A'Hearn, M. F., \& Battams, K. 2013, Icarus, 226, 1350

Lemaire, P., Emerich, C., Vial, J.-C., Curdt, W., Schühle, U., \& Wilhelm, K. 2002a, in ESA Special Publication, Vol. 508, From Solar Min to Max: Half a Solar Cycle with SOHO, ed. A. Wilson, 219-222

Lemaire, P., Emerich, C., Vial, J.-C., Curdt, W., Schühle, U., \& Wilhelm, K. 2002b, in ESA Special Publication, Vol. 508, From Solar Min to Max: Half a Solar Cycle with SOHO, ed. A. Wilson, 219-222

Mäkinen, J. T. T., Bertaux, J., Combi, M. R., \& Quémerais, E. 2001a, Science, 292, 1326
Mäkinen, J. T. T., Bertaux, J., Pulkkinen, T. I., Schmidt, W., Kyrölä, E., Summanen, T., Quémerais, E., \& Lallement, R. 2001b, A\&A, 368, 292

Marsden, B. G. 2005, ARA\&A, 43, 75

McCauley, P. I., Saar, S. H., Raymond, J. C., Ko, Y.-K., \& Saint-Hilaire, P. 2013, ApJ, 768, 161

Noci, G., Kohl, J. L., \& Withbroe, G. L. 1987, ApJ, 315, 706

Noci, G. \& Maccari, L. 1999, A\&A, 341, 275

Opher, M., Bibi, F. A., Toth, G., Richardson, J. D., Izmodenov,

V. V., \& Gombosi, T. I. 2009, Nature, 462, 1036

Oxenius, J. 1965, J. Quant. Spec. Radiat. Transf., 5, 771

Raymond, J. C., Fineschi, S., Smith, P. L., Gardner, L., O'Neal, R., Ciaravella, A., Kohl, J. L., Marsden, B., Williams, G. V., Benna, C., Giordano, S., Noci, G., \& Jewitt, D. 1998, ApJ, 508, 410

Raymond, J. C., McCauley, P. I., Cranmer, S. R., \& Downs, C. 2014, ApJ, 788, 152

Raymond, J. C., Uzzo, M., Ko, Y., Mancuso, S., Wu, R., Gardner, L., Kohl, J. L., Marsden, B., \& Smith, P. L. 2002, ApJ, 564, 1054

Scholz, T. T. \& Walters, H. R. J. 1991, ApJ, 380, 302

Schultz, D. R., Krstic, P. S., Lee, T. G., \& Raymond, J. C. 2008, ApJ, 678, 950

Sekanina, Z. \& Chodas, P. W. 2004, ApJ, 607, 620

-. 2007, ApJ, 663, 657

Sheeley, Jr., N. R., Wang, Y., Hawley, S. H., Brueckner, G. E. Dere, K. P., Howard, R. A., Koomen, M. J., Korendyke, C. M., Michels, D. J., Paswaters, S. E., Socker, D. G., St. Cyr, O. C., Wang, D., Lamy, P. L., Llebaria, A., Schwenn, R., Simnett,

G. M., Plunkett, S., \& Biesecker, D. A. 1997, ApJ, 484, 472

Strachan, L., Suleiman, R., Panasyuk, A. V., Biesecker, D. A., \& Kohl, J. L. 2002, ApJ, 571, 1008

Uzzo, M. \& Marsden, B. G. 2002, Minor Planet Electronic Circulars, 17

Uzzo, M., Raymond, J. C., Biesecker, D., Marsden, B., Wood, C., Ko, Y., \& Wu, R. 2001, ApJ, 558, 403

Williams, L. L. \& Zank, G. P. 1994, J. Geophys. Res., 99, 19229 